\title{
Influence des interactions entre écrans de soutènement sur le calcul de la butée
}

\author{
Jean-Pierre Magnan ${ }^{1,2}$ et Grégory Meyer ${ }^{2, \star}$ \\ ${ }^{1}$ IFSTTAR, Marne-la-Vallée, France \\ 2 SYSTRA, Paris, France
}

\begin{abstract}
Résumé - La mobilisation de la butée devant un écran implique un volume de sol important, sur une distance plus grande que la fiche et qui dépend des paramètres du calcul. L'article passe en revue les méthodes de calcul utilisées pour évaluer la butée, en insistant sur la distance nécessaire au libre développement du mécanisme de butée. Il évalue ensuite de différentes façons l'effet de l'interaction entre deux écrans placés face à face de part et d'autre d'une excavation. La méthode recommandée pour calculer la butée mobilisable consiste à faire un calcul en éléments finis avec des valeurs réduites des paramètres de résistance au cisaillement dans la zone où se développera la butée. Cette démarche permet de déterminer des facteurs correctifs à appliquer au calcul de la butée d'un écran isolé en fonction du rapport de la distance entre écrans à leur fiche.
\end{abstract}

Mots clés : écrans de soutènement / interaction / butée / méthode des éléments finis

\begin{abstract}
Influence of embedded wall interactions on passive earth pressure calculation. The development of passive resistance at the toe of an embedded wall needs an important volume of soil, on a distance larger that the embedded part of the wall, which depends on the calculation data. The paper reviews the calculation methods used to estimate the passive pressure and insists on the distance needed for the full development of the soil resistance. Then, if calculates by different methods the effect of the interaction of two parallel embedded walls placed on opposite sides of an excavation. The recommended method for evaluating the available passive pressure consists in a finite element analysis, using reduced values of the strength parameters in the zone where passive pressure will develop. This procedure gives the possibility to determine correction factors to be applied to the passive pressure calculated for an isolated wall, as a function of the ratio of the walls distance to their embedment depth.
\end{abstract}

Keywords: embedded walls / interaction / passive earth pressure / finite element method

\section{Introduction}

La conception des ouvrages du nouveau réseau urbain du Grand Paris a remis au premier plan certaines problématiques connues depuis longtemps, mais dont les enjeux nécessitent un approfondissement des méthodes de calcul, faute de quoi certains ouvrages ne sont pas raisonnablement dimensionnés. C'est le cas du calcul de la résistance du sol (butée) entre des écrans en vis-à-vis proche, qui est directement influencé par la distance entre ces écrans. L'étude présentée dans cet article s'inspire d'un cas réel rencontré sur la ligne 15 Sud du Grand Paris Express (tronçon T2), sur une gare ancrée dans des terrains relativement mous en comparaison des horizons adjacents (Calcaire Grossier au-dessus et Craie en dessous), à

\footnotetext{
$\bar{\star}$ Auteur de correspondance : gmeyer@systra.com
}

savoir les Argiles plastiques. Le coefficient de butée d'un tel sol devant un écran isolé est relativement faible et nécessiterait un approfondissement des parois dans la Craie, à plus de $20 \mathrm{~m}$ sous l'argile. Nous montrons que la présence d'un écran proche permet de justifier la stabilité de l'ouvrage et proposons une méthodologie de calcul pour y parvenir, par intégration dans un calcul MISS ou dans un calcul aux éléments finis.

La poussée et la butée font partie, avec la portance du sol et les instabilités de pentes, des mécanismes de rupture des massifs de sols. Ces ruptures correspondent à des mécanismes de déformation qui doivent respecter les conditions aux limites du massif (et de la structure) et aboutissent à des cinématiques de rupture lorsque les résistances cessent d'équilibrer les actions.

Le calcul de stabilité (donc aussi le calcul de la poussée et de la butée) a pour but de définir cette limite. Depuis une cinquantaine d'années, les théoriciens de la mécanique ont 

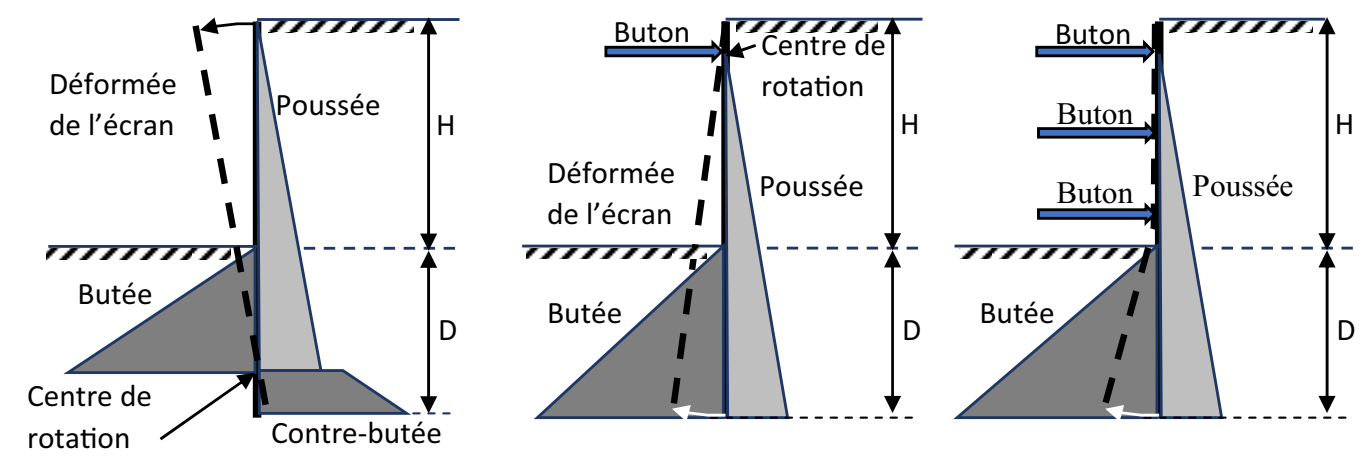

Fig. 1. Différentes situations de calcul de butée.

Fig. 1. Different situations for passive earth pressures calculations.

privilégié une description énergétique, dans laquelle on compare le travail des forces provoquant la rupture au travail des forces (ou contraintes) résistant à la rupture et l'on peut chercher la limite en partant d'états stables (approche par l'intérieur) ou bien en partant d'états instables (approche par l'extérieur). Les différences entre méthodes viennent des hypothèses faites pour le calcul.

Le calcul de la butée du sol ne peut être isolé du calcul du fonctionnement de l'écran. La première étape de l'étude de la résistance en butée est de définir dans quelles zones du terrain elle va être mobilisée. On distingue ainsi (Fig. 1):

- les écrans en console, avec des zones en butée et en contrebutée;

- les écrans avec un point d'appui (en haut par buton ou tirant, ou en bas par un radier);

- les écrans à appuis multiples (au moins deux).

Les méthodes de calcul recommandées actuellement (norme européenne NF EN 1997-1 ou Eurocode 7, norme française NF P94-282 sur le calcul des écrans, norme d'application de l'Eurocode 7) incluent deux approches: le calcul de l'instabilité du massif (MEL), applicable aux écrans en console et à un point d'appui, et le calcul de l'interaction entre le sol et la structure (MISS), qui permet de traiter le cas des écrans à appuis multiples.

Ces calculs peuvent être effectués par des méthodes analytiques (modèles d'équilibre de blocs ou de champs de contraintes pour l'équilibre limite, méthodes de type «poutre sur appuis élastiques » pour les écrans à appuis multiples) ou par des méthodes numériques, notamment par la méthode des éléments finis avec des modèles élastoplastiques.

Les méthodes de calcul classiques de l'équilibre limite en butée ont été développées pour les écrans isolés, sans vis-à-vis. Pour cette raison elles ne sont pas toujours applicables, mais les limites d'application de ces méthodes, qui sont liées aux dimensions du volume de sol impliqué dans la résistance du massif, ne sont pas clairement identifiées. Le présent article a pour objectif d'identifier ces limites et de proposer les corrections nécessaires en cas d'interaction entre écrans. Il est utile de rappeler que la force de butée est définie comme l'action de l'écran sur le sol et qu'il faut utiliser la force opposée pour calculer l'écran.

\section{Les méthodes classiques d'évaluation de la butée}

L'analyse de la butée (et de la poussée) commence à l'époque de Coulomb ( $18^{\mathrm{e}}$ siècle) mais elle a été développée ultérieurement par de nombreux auteurs, avec une évolution vers les méthodes de résolution dite de statique graphique dérivées des calculs d'équilibre de solides introduits par Coulomb. L'accès à ces documents anciens n'est pas toujours aisé, mais la numérisation des livres anciens offre désormais un accès plus facile. Le «blog» de Pierre Montès (Montréal), accessible en 2017, mis à jour le 17 avril 2012, donne des liens vers plusieurs des ouvrages scannés qui ont marqué l'évolution des calculs de butée depuis Coulomb: Coulomb (1773), numérisé par la Bibliothèque Nationale de France (Gallica) comme celui de Résal (1903); Poncelet (1840), Bresse (1859), Culmann (1866), Rebhann (1871), Müller-Breslau (1881), scannés par Google. Montès cite aussi une revue critique de ces méthodes de calcul par Corradi (1995). Parmi les ouvrages plus récents, le livre de Caquot (1934) a été scanné par l'École Nationale des Ponts et Chaussées.

\subsection{Analyse de l'équilibre de blocs}

La méthode proposée par Coulomb (1773) s'appuie sur la seule mécanique existant à cette époque, la mécanique des solides, avec des forces et des déplacements et des conditions de glissement entre ces solides. La méthode a été établie sur la base de l'observation des coins en glissement derrière les murs de soutènement et avec une orientation quelconque de la force de poussée ou butée. Elle permet de traiter la configuration représentée sur la figure 2 , avec comme seule contrainte que le bloc qui glisse ait la forme d'un prisme, dans un massif homogène. La théorie de Coulomb a été établie pour un parement vertical $(\lambda=0)$ et un massif à surface horizontale. L'extension au cas d'un parement incliné et d'une surface inclinée $(\beta>0)$ est due à Poncelet (1840). Les angles $\lambda$ et $\beta$, comptés à partir de la direction de référence (verticale ou horizontale) sont positifs dans le sens trigonométrique.

La force de poussée $\mathrm{Q}_{\mathrm{a}}$, inclinée de l'angle $\delta_{\mathrm{a}}$ par rapport à la normale au parement, est donnée par l'équation (1), obtenue par maximisation par rapport aux valeurs de l'angle $\theta$. 


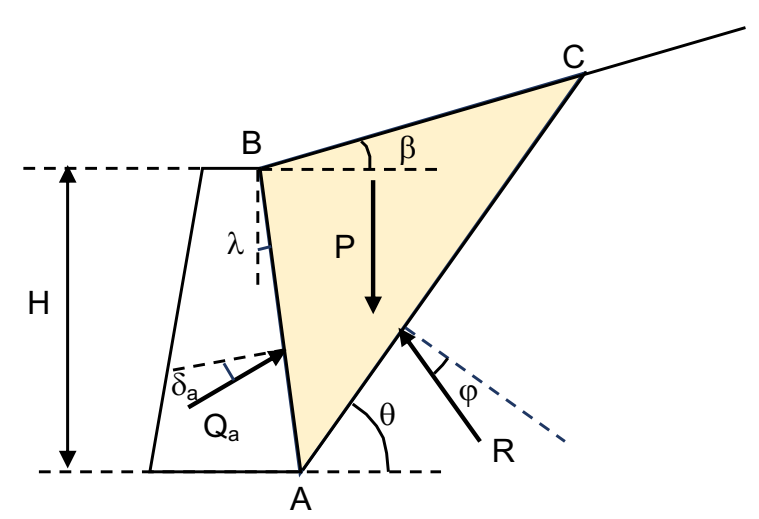

Fig. 2. Schéma de calcul de Coulomb pour la poussée sur un mur de hauteur $\mathrm{H}$.

Notations: $\beta$ : pente du talus du terrain derrière le mur; $\delta_{a}$ : inclinaison de la force de poussée $\mathrm{Q}_{\mathrm{a}}$ par rapport à la normale au mur $(0$ à $\varphi) ; \lambda$ : inclinaison du parement arrière du mur par rapport à la verticale ; $\theta$ : paramètre de calcul (inclinaison de la base $\mathrm{AC}$ du prisme $\mathrm{ABC})$; $\mathrm{P}$ : poids du prisme $\mathrm{ABC}$; $\mathrm{R}$ : force de frottement limite sur $\mathrm{AC}$ (inclinée de $\varphi$ par rapport à la normale).

Fig. 2. Coulomb's design diagram for calculating active earth pressure on a wall with height $\mathrm{H}$.

Notations: $\beta$ : slope of the ground behind the wall; $\delta_{\mathrm{a}}$ : inclination of the active force $Q_{a}$ with respect to the normal to the wall $(0$ to $\varphi) ; \lambda$ : inclination of the rear face of the wall with respect to vertical; $\theta$ : angle used for calculations (inclination of the base $\mathrm{AC}$ of prism $\mathrm{ABC}$ ); $\mathrm{P}$ : weight of prism $\mathrm{ABC}$; $\mathrm{R}$ : limit friction force acting on $\mathrm{AC}$ (inclined to the normal by $\varphi$ ).

$$
\begin{aligned}
\mathrm{Q}_{\mathrm{a}} & =\frac{1}{2} \gamma \mathrm{H}^{2} \mathrm{~K}_{\mathrm{a}} \\
& =\frac{1}{2} \gamma \mathrm{H}^{2} \frac{\cos ^{2}(\varphi-\lambda)}{\cos ^{2} \lambda \cos \left(\lambda+\delta_{a}\right)}\left[1+\sqrt{\frac{\sin \left(\varphi+\delta_{a}\right) \sin (\varphi-\beta)}{\cos \left(\lambda+\delta_{a}\right) \cos (\lambda-\beta)}}\right]^{-2} .
\end{aligned}
$$

La valeur de la force de poussée a été validée par la pratique.

La méthode adoptée par Coulomb pour calculer la poussée du terrain sur un mur de soutènement a été appliquée au problème « inverse », dans lequel le mur appuie sur le terrain (sol en conditions de butée), mais sans avoir de validation expérimentale parce que l'on ne mobilisait pas de butée à cette époque.

Les calculs de butée s'effectuent selon le schéma représenté sur la figure 3 .

La force de butée $\mathrm{Q}_{\mathrm{p}}$, inclinée de l'angle $\delta_{\mathrm{p}}$ par rapport à la normale au parement, est donnée par l'équation (2), obtenue par minimisation par rapport aux valeurs de l'angle $\theta$.

$$
\begin{aligned}
\mathrm{Q}_{\mathrm{p}} & =\frac{1}{2} \gamma \mathrm{H}^{2} \mathrm{~K}_{\mathrm{p}} \\
& =\frac{1}{2} \gamma \mathrm{H}^{2} \frac{\cos ^{2}(\varphi+\lambda)}{\cos ^{2} \lambda \cos \left(\lambda-\delta_{p}\right)}\left[1+\sqrt{\frac{\sin \left(\varphi+\delta_{p}\right) \sin (\varphi+\beta)}{\cos \left(\lambda-\delta_{p}\right) \cos (\lambda-\beta)}}\right]^{-2} .
\end{aligned}
$$

Cette valeur de la force de butée a été contestée quand on a commencé à créer des rideaux de soutènement mobilisant la

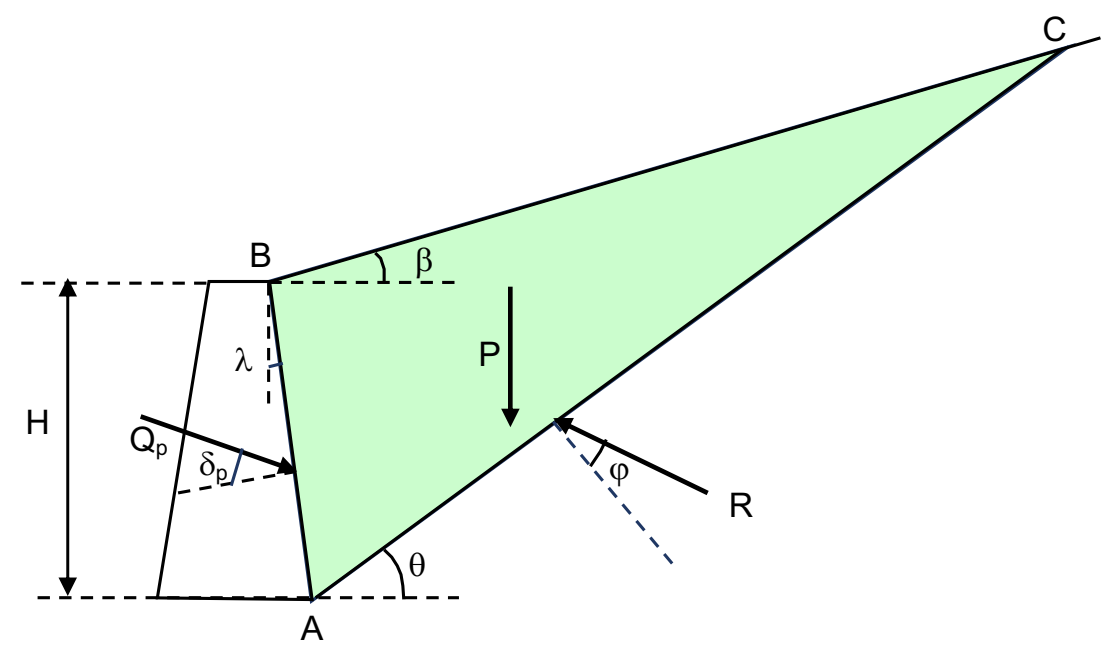

Fig. 3. Schéma de calcul de Coulomb pour la butée sur un mur de hauteur $H$.

Notations : $\beta$ : pente du talus du terrain derrière le mur; $\delta_{\mathrm{p}}$ : inclinaison de la force de poussée $\mathrm{Q}_{\mathrm{p}}$ par rapport à la normale au mur $(-\varphi$ à 0$) ; \lambda$ : inclinaison du parement arrière du mur par rapport à la verticale; $\theta$ : paramètre de calcul (inclinaison de la base $\mathrm{AC}$ du prisme $\mathrm{ABC}$ ); $\mathrm{P}:$ poids du prisme $\mathrm{ABC} ; \mathrm{R}$ : force de frottement limite sur $\mathrm{AC}$ (inclinée de $\varphi$ par rapport à la normale).

Fig. 3. Coulomb's design diagram for calculating passive earth pressure on a wall with height $\mathrm{H}$.

Notations: $\beta$ : slope of the ground behind the wall; $\delta_{\mathrm{p}}$ : inclination of the passive force $\mathrm{Q}_{\mathrm{p}}$ with respect to the normal to the wall $(-\varphi$ to 0$) ; \lambda$ : inclination of the rear face of the wall with respect to vertical; $\theta$ : angle used for calculations (inclination of the base AC of prism ABC); $\mathrm{P}$ : weight of prism $\mathrm{ABC}$; R: limit friction force acting on $\mathrm{AC}$ (inclined to the normal by $\varphi$ ). 
butée à la fin du $19^{\mathrm{e}}$ siècle et elle a été remplacée ultérieurement par la solution de Caquot, appelée aussi méthode de la spirale logarithmique.

La valeur attribuée à l'inclinaison $\delta$ de la force de poussée ou de butée est un choix lié aux conditions de frottement du sol contre l'écran (au maximum l'angle de frottement interne $\varphi \mathrm{du}$ sol) et son sens est lié au déplacement relatif de l'écran (le parement du mur) par rapport au sol. Si le prisme de sol glisse vers le bas (poussée) la force de contact exercée par l'écran sur le sol est dirigée vers le haut (et inversement pour la butée).

\subsection{Analyse de l'équilibre des contraintes}

Le développement de la mécanique des milieux continus et l'introduction du cercle de Mohr au cours du $19^{\mathrm{e}}$ siècle ont permis de modifier l'analyse de l'équilibre des massifs de sols.

Par analogie avec l'équilibre de blocs rigides de la méthode de Coulomb, qui permet de définir la force de contact avec l'écran sans prendre en compte explicitement les déplacements du sol, les travaux ont visé à analyser l'équilibre de champs de contraintes sans tenir compte des champs de déformations. Il s'agit de construire des champs de contraintes qui correspondent aux conditions limites d'équilibre du sol. Les travaux de Rankine (1857), puis Boussinesq (1876), Résal, etc. et enfin Caquot, qui a publié en 1934 la méthode utilisée pour établir les abaques de Caquot-Kérisel recommandés dans l'Eurocode 7 et ses normes d'application, illustrent différentes voies pour obtenir de tels champs de contraintes. Absi (1984) a repris et complété les études antérieures sur l'utilisation de la théorie de la plasticité pour évaluer les équilibres limites dans les massifs de sols.

L'outil de base de la construction de ces champs de contraintes est la définition des deux plans qui correspondent aux conditions de rupture sur le cercle de Mohr. Dans le cas d'un sol dont la résistance est caractérisée par un angle de frottement interne et une cohésion nulle, les deux plans (1 et 2) sur lesquels s'exercent les contraintes de rupture ont des orientations conjuguées (Fig. 4), c'est-à-dire que le vecteur contrainte qui s'exerce sur l'un des plans est parallèle à la trace de l'autre plan et réciproquement. Rappelons que, quand le plan tourne d'un angle $\theta$, le point représentant la contrainte appliquée sur ce plan tourne de $-2 \theta$ sur le cercle de Mohr.

Si l'on construit à partir de l'écran les contraintes conjuguées à la rupture, ce qui représente aussi les surfaces sur lesquelles ces contraintes s'appliquent, on trouve des réseaux de lignes de glissement tels que ceux représentés sur la figure 5. Sur cette figure, les deux schémas du haut représentent le mécanisme de glissement imaginé par Rankine (poussée à gauche, butée à droite). Les quatre schémas suivants correspondent à différents cas de lignes de rupture associées à la théorie de Caquot pour différentes valeurs de l'angle $\delta$ (de même, poussée à gauche, butée à droite).

Le choix de la valeur de l'inclinaison $\delta$ de la force de poussée ou de butée par rapport à la normale à la surface de l'écran en contact avec le sol doit être fait en fonction du déplacement relatif du sol et de l'écran. Si l'écran s'enfonce, la force de contact exercée par l'écran sur le sol est dirigée vers le bas. Si le sol s'enfonce par rapport à l'écran, la force de contact est dirigée vers le haut. Le choix de l'orientation de $\varphi$ doit donc
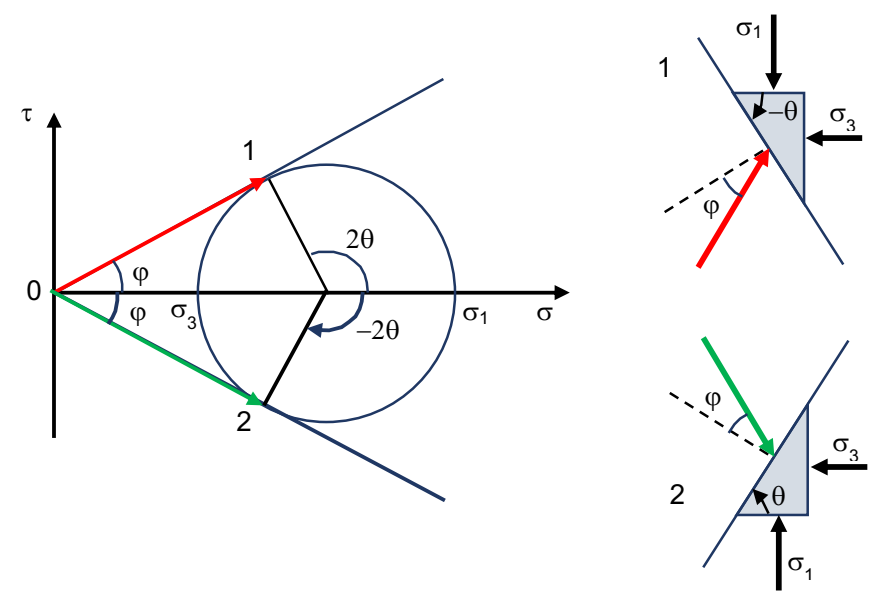

Fig. 4. Position des contraintes conjuguées sur le cercle de Mohr. Fig. 4. Position of conjugate stresses on Mohr's circle.

Tableau 1. Distance d'influence de la butée pour un écran de fiche $\mathrm{D}=10 \mathrm{~m}$ (Schéma de Coulomb)

Table 1. Distance of influence of passive pressures for an embedded wall with $\mathrm{D}=10 \mathrm{~m}$ (Coulomb's scheme).

\begin{tabular}{llllllll}
\hline Angle $\varphi$ (degrés) & 0 & 20 & 25 & 30 & 35 & 40 & 45 \\
Distance d (m) & 10 & 14,3 & 15,7 & 17,3 & 19,2 & 21,4 & 24,1 \\
\hline
\end{tabular}

être analysé dans chaque cas en fonction des déplacements avant la rupture.

Les équations différentielles du modèle de calcul de Boussinesq ne peuvent être résolues sous forme analytique, mais Caquot a proposé une méthode de résolution approchée qui a produit des tables de valeurs numériques (Kérisel et Absi, 2003), que l'on peut aussi représenter sous forme graphique, comme sur la figure 6 (d'après le manuel NAVFAC DM 7-2, US Department of the Navy, 1982) ou dans l'annexe C de l'Eurocode 7.

\subsection{Volume de sol mobilisé par la butée}

La contrainte de butée au contact de l'écran n'est pas une propriété locale du massif, mais provient de la mobilisation de la résistance au cisaillement dans un certain volume de sol devant la fiche de l'écran. On peut avoir une idée de la distance nécessaire en observant que, dans le modèle de Coulomb, cette distance vaut $\mathrm{d}=\mathrm{D} / \tan (\pi / 4-\varphi / 2)$, en désignant par $\mathrm{D}$ la fiche de l'écran. Pour $\mathrm{D}=10 \mathrm{~m}$ et des angles de frottement interne $\varphi$ croissants, on obtient les valeurs du tableau 1 .

Le schéma de calcul de Caquot va produire une distance d'influence différente, plus faible si le frottement est dirigé vers le haut (le sol tasse par rapport à l'écran) et plus grande si le frottement est dirigé vers le bas (l'écran tasse par rapport au sol, ce qui est le cas le plus courant). Dans le cas représenté en bas à droite de la figure 5, dessiné pour un angle de frottement interne de 37 degrés, la distance d'influence d'un écran de $10 \mathrm{~m}$ de fiche est égale à $32,5 \mathrm{~m}$ (au lieu de 20,1 $\mathrm{m}$ dans le schéma de Coulomb pour $\delta=0$ ). 

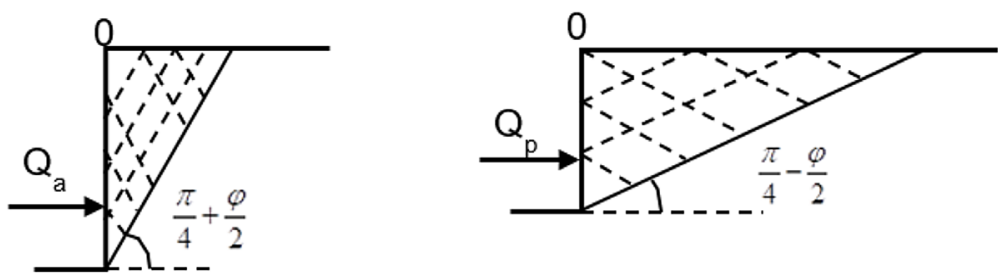

RANKINE $(\delta=0)$
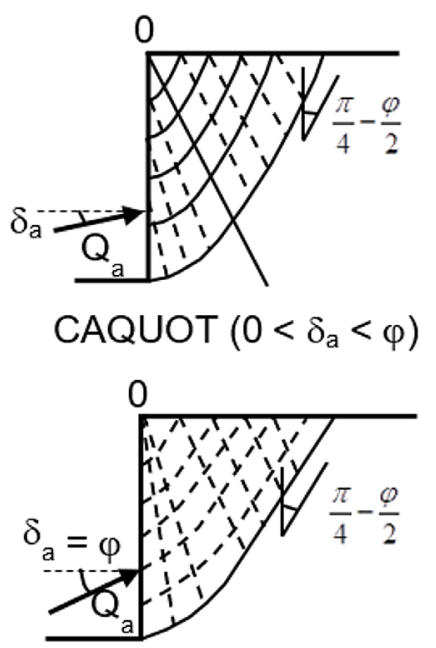

$$
\text { CAQUOT }\left(\delta_{\mathrm{a}}=\varphi\right)
$$

\section{a) Équilibre limite de poussée}

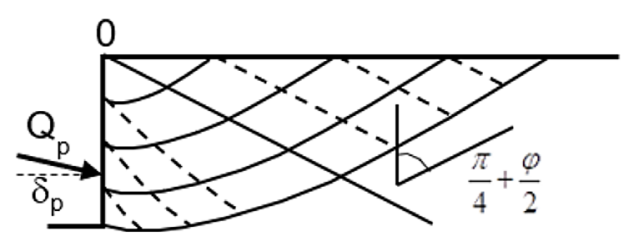

CAQUOT $\left(0<\delta_{\mathrm{p}}<\varphi\right)$

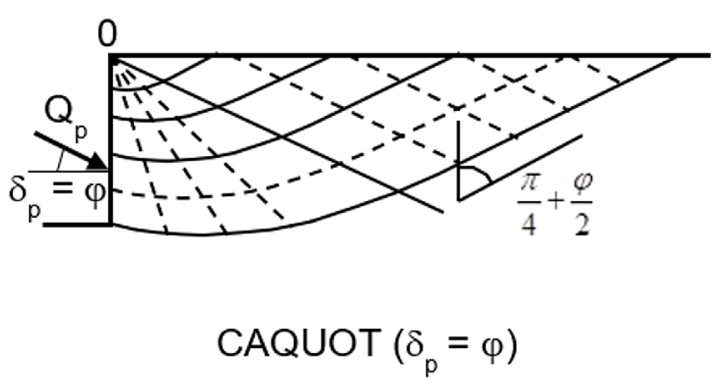

b) Équilibre limite de butée

Fig. 5. Réseaux de lignes de glissement pour les calculs de poussée et butée.

(d'après Philipponnat et Hubert, 1997).

Fig. 5. Slip-lines fields for calculating active and passive pressures.

(after Philipponnat and Hubert, 1997).

La question de l'interaction des écrans en vis-à-vis est donc importante et peut concerner de nombreuses fouilles profondes, comme celles des ouvrages du futur métro parisien et des constructions semblables. Les principes généraux de la mécanique permettent de prévoir que la résistance augmentera si l'espace nécessaire n'est pas fourni au mécanisme de butée. C'est ce que l'on vérifiera dans la deuxième partie de cet article.

\subsection{Analyse de l'équilibre des contraintes en tenant compte des déformations}

Le défaut des théories d'équilibre des contraintes est qu'elles fonctionnent indépendamment des déformations des sols, et notamment des obstacles qui peuvent perturber les mécanismes de rupture. En particulier, elles ne peuvent traiter simultanément les mécanismes de poussée et de butée, ni analyser l'interaction de deux rideaux situés face à face.

Intégrer les déformations du sol dans le calcul des efforts appliqués aux soutènements peut se faire de façon locale, avec des coefficients de réaction qui représentent l'interaction entre le mouvement de l'écran et la pression de contact avec le sol et permettent de calculer une structure «sur appuis élastiques (à pression de contact limitée)». Ce type de calcul permet d'évaluer la déformation de la structure et les efforts internes nécessaires pour dimensionner l'écran. La norme française NF P94-282 l'appelle «Modèle d'interaction sol-structure ou MISS - Modèle aux coefficients de réaction » et le recommande pour le calcul des écrans à appuis multiples. Cette limitation est liée au fait que les déplacements d'ensemble ne sont pas correctement représentés dans ce modèle de calcul, qui fonctionne bien pour des appuis fixes ou légèrement déformables, comme le calcul des poutres sur appuis élastiques.

L'interaction locale du terrain et de l'écran est représentée par une relation telle que celle de la figure 7 , où $\mathrm{H}$ est la hauteur libre de l'écran.

Ce modèle de calcul est utilisé dans les programmes tels que RIDO, KREA, PARIS, etc. Il est combiné avec les propriétés de l'écran considéré comme une poutre, et des appuis localisés des butons et dalles en contact avec l'écran. Mais il ne tient pas compte des conditions géométriques générales du projet (profondeur du substratum sous le pied de l'écran, présence d'un autre écran en vis-à-vis).

En fin de calcul, on vérifie que les pressions sur l'écran, du côté de la fouille, sont inférieures à la butée mobilisable dans le terrain. Cette vérification doit être faite en tenant compte de la 

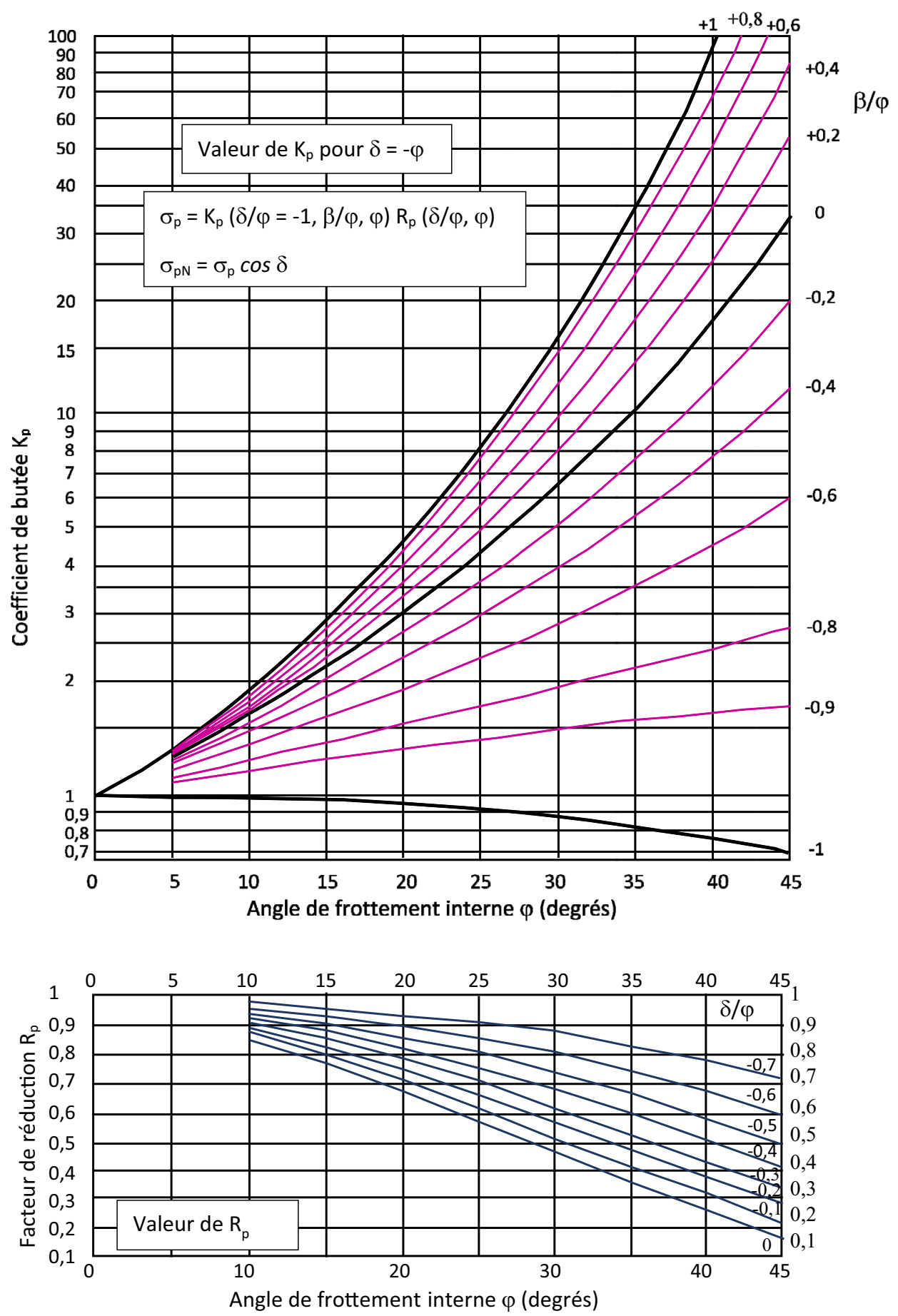

Fig. 6. Coefficients de butée (d'après NAVFAC DM-7.2, 1982).

Fig. 6. Coefficients of passive pressures (after NAVFAC DM-7.2, 1982).

géométrie du site pour l'évaluation de la butée. En cas de parois parallèles proches, cette condition n'est pas respectée par le modèle de Caquot.

\section{Calculs par la méthode des éléments finis}

La résolution des équations de la mécanique des milieux continus permet d'obtenir à la fois les déformations et les contraintes dans la situation d'équilibre. On peut tenir compte des conditions géométriques du projet et donc échapper à certaines limitations des méthodes précédentes. Cependant, ce type de calcul demande des informations plus complètes sur le comportement du sol, de l'écran et des appuis, et la précision du résultat est liée au maillage utilisé (type et dimension des éléments, extension verticale et horizontale du maillage, nécessité de transformer les appuis localisés comme les butons pour les calculs bidimensionnels). Si le maillage est 


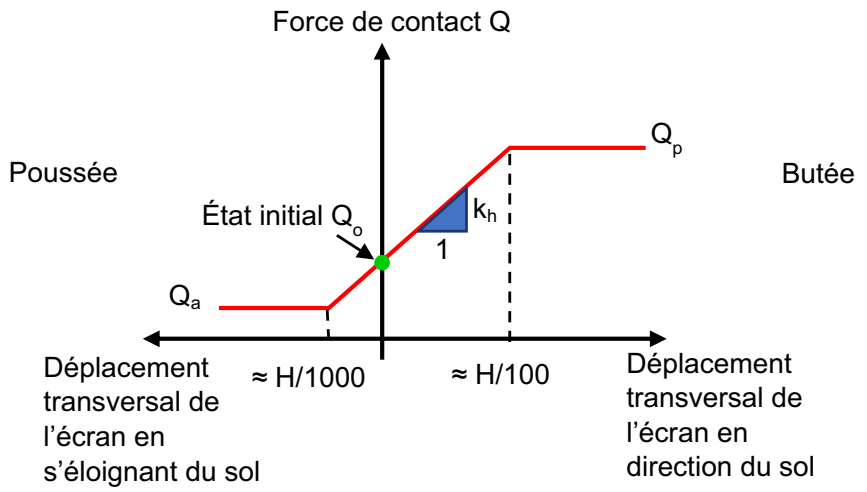

Fig. 7. Schéma d'interaction locale avec coefficient de réaction $k_{h}$. Fig. 7. Local interaction model with coefficient of subgrade reaction $k_{h}$.

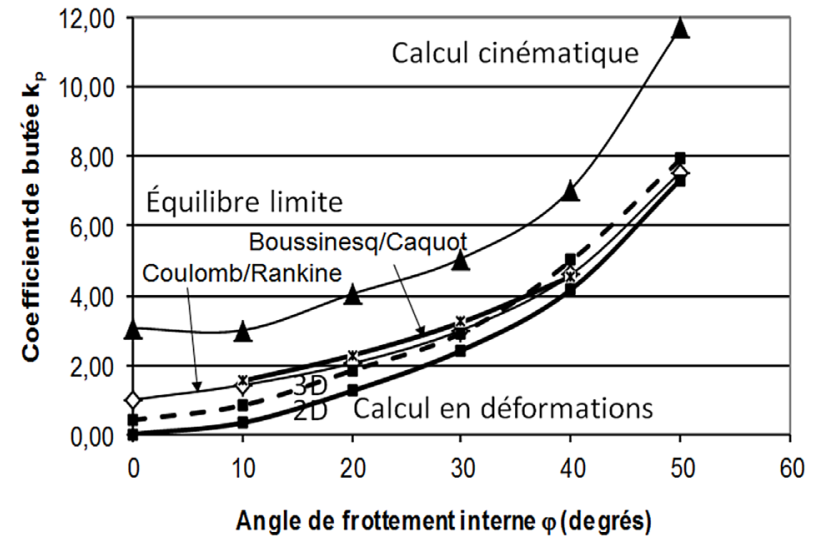

Fig. 9. Comparaison des calculs de $\mathrm{K}_{\mathrm{p}}$ en déformations, en analyse cinématique et par les méthodes analytiques de Coulomb et Caquot. Fig. 9. Comparison of $\mathrm{K}_{\mathrm{p}}$ calculations by stress-strain analysis, kinematic analysis and analytic methods of Coulomb and Caquot.

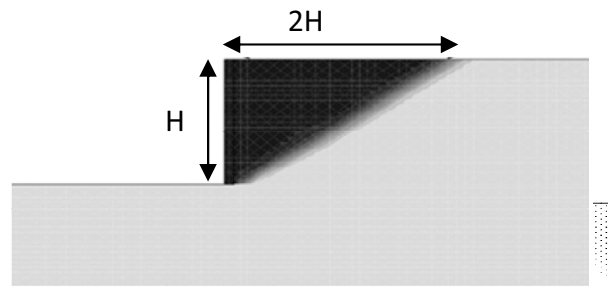

a. Calcul en déformations

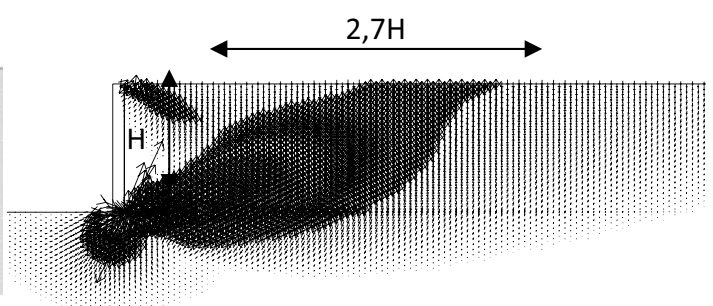

b. Calcul cinématique (vitesses de déformations)

Fig. 8. Calcul de l'état limite par l'intérieur (a) et par l'extérieur (b) pour $\varphi=30$ degrés.

Fig. 8. Determination of limit state for $\varphi=30$ degrees. (a) Lower bound. (b) Upper bound.

suffisamment fin, les champs de contraintes sont quasi continus et on peut les comparer avec ceux des méthodes qui ne considèrent que l'équilibre des contraintes (type Caquot), qui sont des approches «par l'intérieur».

Le calcul en éléments finis (ou en différences finies pour les logiciels comme FLAC), a été validé par comparaison avec les solutions analytiques rappelées dans les sections précédentes. Il peut être appliqué au calcul de l'équilibre limite (MEL) comme au calcul d'interaction sol-structure (MISS). L'étude numérique de la poussée et de la butée des terres a fait l'objet de beaucoup de publications au cours des dernières années, comme celle de Mokhbi et Messaset (2014) pour des écrans isolés.

Les travaux effectués au LCPC (devenu IFSTTAR) au début des années 2000 ont appliqué au calcul de soutènements une approche de calcul à la rupture "par l'extérieur», qui consiste à construire des champs de vitesses de rupture respectant les conditions de chargement et aux limites, mais dépassant la résistance des matériaux.

Pour les calculs d'équilibre limite, on obtient des résultats tels que ceux de la figure 8 , où l'on montre un calcul élastoplastique (approche par l'intérieur) et un calcul cinématique de rupture (approche par l'extérieur) réalisés avec CésarLCPC (Droniuc et Magnan, 2010). Les mécanismes de rupture sont peu différents de ceux des méthodes traditionnelles. L'approche cinématique donne une valeur plus forte de la butée, que l'on peut réduire en affinant le calcul (Fig. 9). L'étendue du mécanisme de butée (Fig. 10) est toujours plus importante que celle déduite du modèle de Coulomb.

La méthode des éléments finis, mise en œuvre au moyen de Plaxis, est utilisée dans la suite de cet article pour caractériser l'interaction des mécanismes de butée lorsque deux écrans sont face à face, en fonction de leur distance.

\section{Le contexte normatif et les limites des abaques de Caquot-Kérisel-Absi}

La norme française NF P94-282 sur le calcul des écrans indique ( $\S$ 5.1.3.4 Valeurs limites de la pression des terres) que «Il convient de déterminer les valeurs de la poussée et de la butée des terres sous l'effet du poids volumique, d'une surcharge uniforme q et de la cohésion, à partir des coefficients proposés par Kérisel et Absi (voir Notes 1 à 3) lorsque c'est approprié (voir Note 4).»

La Note 2 indique que «Il est admis de déterminer les coefficients de poussée-butée des terres à partir d'une procédure numérique du côté de la sécurité, comme par exemple celle indiquée en Annexe C.2 de la norme NF EN 1997-1 (Eurocode 7). ». Toutefois, cette procédure ne concerne que le cas de l'écran isolé.

La Note 4 indique que, dans certains cas (le cas de deux écrans face à face n'est néanmoins pas cité), on peut être amené 


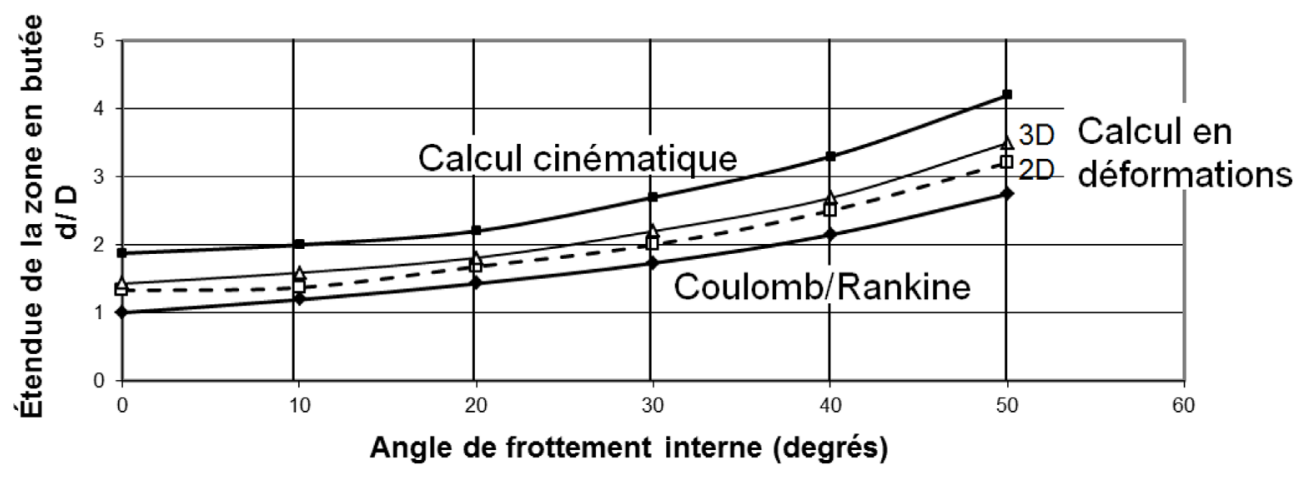

Fig. 10. Étendue d de la zone concernée par le mécanisme de butée, en fonction de la fiche D.

Fig. 10. Extent d of passive earth pressure mechanism, as a function of embedment D.
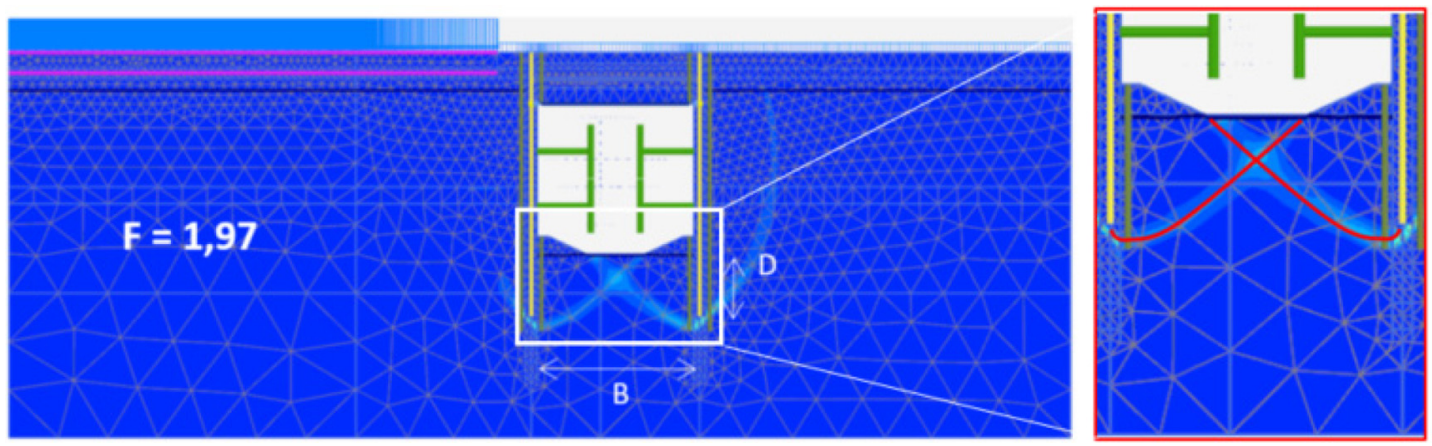

Fig. 11. Mécanismes de rupture en butée de pied de deux écrans opposés proches.

Fig. 11. Passive earth failure mechanisms for two nearby embedded walls.

à appliquer d'autres méthodes, selon les règles données à la suite. En particulier, l'article (4) de la norme indique que: «Lorsque l'on détermine les valeurs limites de la pression des terres sur un écran à partir d'une méthode qui n'est pas fondée sur les coefficients de pression des terres de Kérisel et Absi on doit vérifier que les hypothèses sur lesquelles elle repose sont satisfaites et que les valeurs obtenues s'avèrent proches des valeurs proposées par Kérisel et Absi pour des cas comparables (voir Note 1).»

La référence à Kérisel et Absi (2003) correspond à la $3^{\mathrm{e}}$ édition des abaques de Caquot et Kérisel.

$\mathrm{La}$ réflexion à l'origine du présent article est partie du problème posé par l'évaluation de la butée par les abaques de Caquot-Kérisel-Absi dans le cas d'une excavation entre deux écrans parallèles. Ce cas est brièvement évoqué dans la norme NF P94-282 (Annexe F) pour indiquer que les pratiques liées à un écran isolé sous-estiment le coefficient de réaction lorsque la largeur de l'excavation devant l'écran est inférieure à trois fois la longueur de transfert élastique $1_{0}$ de l'écran. La formule utilisée dans les logiciels de calcul de type RIDO est celle des abaques de Caquot-Kérisel-Absi et est donc limitée au domaine de validité de cette approche.

\section{Effet de la présence de deux rideaux sur la butée}

Dans le cadre des études de la Ligne 15 Sud du GrandParis, le problème de l'interaction des butées de deux écrans

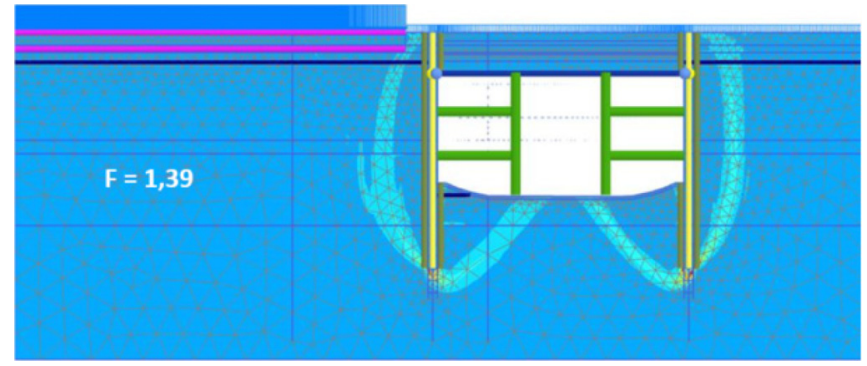

Fig. 12. Mécanismes de rupture en butée de pied de deux écrans opposés éloignés.

Fig. 12. Passive earth failure mechanisms for two distant embedded walls.

parallèles opposés a été rencontré, car les terrains relativement mous en butée obligeaient à approfondir les parois moulées de $20 \mathrm{~m}$ quand le calcul de la butée était réalisé pour un écran isolé. Des calculs réalisés avec le logiciel Plaxis, selon la procédure de «réduction de $\mathrm{c}$ et phi » ont montré (Fig. 11) que les mécanismes de déformation du sol devant les deux écrans dépendent de la distance entre les écrans. L'effort nécessaire pour arriver à la rupture du sol par butée est augmenté par rapport à celui d'une paroi isolée dans la même configuration. Le facteur de sécurité (déduit de la réduction de c et $\varphi$ ) entre un double rideau (Fig. 11) et un écran simple (Fig. 12) est augmenté de $30 \%$ dans cet exemple. 


\section{Analyse de l'interaction des butées de pied de deux écrans opposés}

L'augmentation de la butée du fait de l'intersection des mécanismes de déformation peut être analysée à l'aide de logiciels de type Talren (calcul d'équilibre de blocs sur des surfaces de glissement) ou Plaxis (calculs de déformations élastoplastiques en éléments finis).

Pour ces calculs, on définit un chargement sur l'écran qui a la forme du résultat (rapport constant entre $\sigma^{\prime}{ }_{\mathrm{v}}$ et $\sigma^{\prime}{ }_{\mathrm{h}}$, donc variation linéaire de la pression sur l'écran en fonction de la profondeur et inclinaison constante de la charge par rapport à la normale à l'écran) et on cherche quelle intensité de ce chargement correspond à un mécanisme de rupture.

On a choisi de faire les calculs pour des distances $B$ entre les deux parois égales à $\mathrm{B}=\infty, \mathrm{B}=2 \mathrm{D}, \mathrm{B}=1,5 \mathrm{D}, \mathrm{B}=\mathrm{D}$ et $\mathrm{B}=0,5 \mathrm{D}, \mathrm{D}$ étant la hauteur de fiche de la paroi.

Les calculs sont réalisés ici en condition hors nappe, la pression de l'eau étant ajoutée dans un calcul classique.

\subsection{Calculs à la rupture au moyen de Talren}

L'intérêt d'utiliser Talren est que la modélisation est plus rapide et que les surcharges sont introduites directement avec la valeur de la butée et son angle. On peut remarquer pour un cas simple $\left(c^{\prime}=0 \mathrm{kPa}, \varphi^{\prime}=30\right.$ degrés et $\left.\delta_{\mathrm{p}} / \varphi^{\prime}=0\right)$ que l'on retrouve bien un coefficient de butée $K_{P}$ de 3 et une rupture de type « coin de Coulomb» (Fig. 13). Le fait de borner la rupture à $\mathrm{B}=\mathrm{D}$ (modélisé en demi-largeur sous Talren) montre une augmentation du $K_{P}$ à 3,5 , avec une rupture plus complexe en spirale logarithmique (Fig. 14).

L'introduction d'une inclinaison de la butée à $-2 / 3$ de $\varphi^{\prime}$ modifie la rupture, comme cela a été montré dans la théorie de Caquot et les abaques de Caquot-Kérisel-Absi, pour une paroi isolée (Fig. 15).

Néanmoins, comme Talren ne permet pas de travailler avec deux modes de chargement opposés en même temps, la géométrie de la zone en butée doit forcément être bornée par symétrie. Il s'ensuit que la rupture sous Talren n'est pas rigoureusement acceptable dans le cas d'une fouille de largeur limitée (Fig. 16).

Les calculs en éléments finis (logiciel Plaxis) ont donc été préférés pour la suite de cette étude.

\subsection{Calculs en déformations au moyen de Plaxis}

Dans les calculs présentés ci-après, le sol est modélisé par une loi de comportement élastoplastique (élasticité linéaire isotrope et critère de rupture de Mohr-Coulomb). Les paramètres de résistance valent $\mathrm{c}^{\prime}=0 \mathrm{kPa}, \varphi^{\prime}=30$ degrés. Le module d'élasticité vaut $\mathrm{E}=60 \mathrm{MPa}$ et le coefficient de Poisson $v=0,3$. Il n'y a pas d'eau.

S'agissant d'une étude de type paramétrique, le calcul est effectué de la façon suivante:

- création du maillage total et initialisation des contraintes;

- excavation du terrain derrière l'écran, application d'une pression équivalente aux contraintes horizontales sur l'écran et application d'une pression équivalente sur la

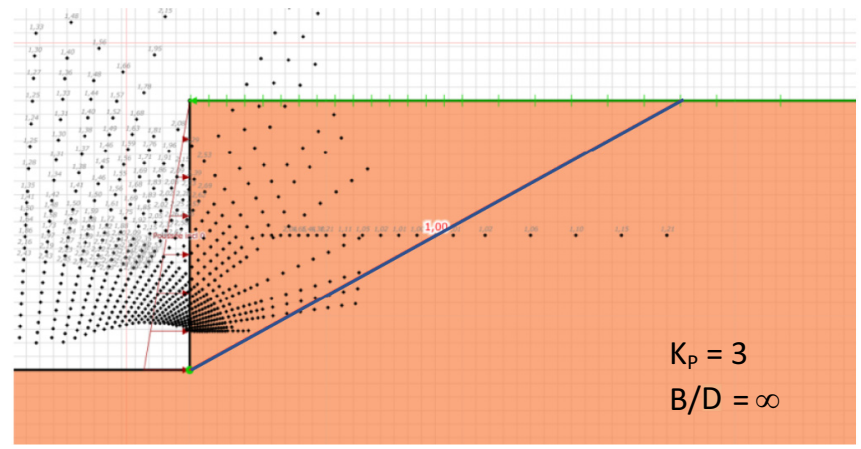

Fig. 13. Calcul de stabilité avec Talren (glissement de bloc rigide). Une paroi isolée.

Fig. 13. Stability analysis using Talren (stability of a rigid block). Isolated embedded wall.

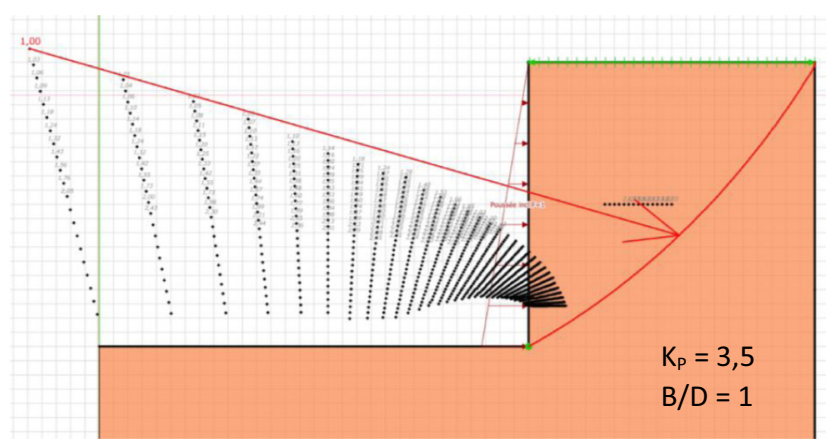

Fig. 14. Calcul de stabilité avec Talren (glissement de bloc rigide). Deux parois en vis-à-vis.

Fig. 14. Stability analysis using Talren (stability of a rigid block). Two parallel embedded walls.

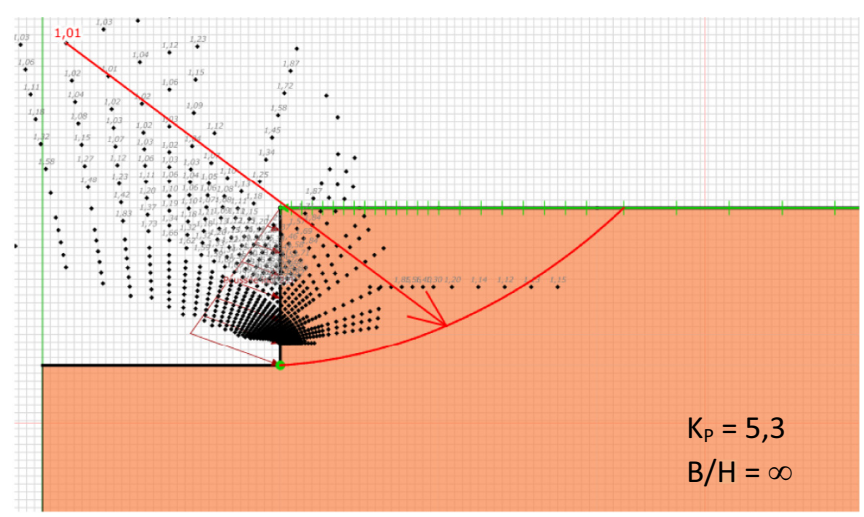

Fig. 15. Calcul de stabilité avec Talren (glissement de bloc rigide). Paroi isolée. $\delta=-2 \varphi^{\prime} / 3$.

Fig. 15. Stability analysis using Talren (stability of a rigid block). Isolated embedded wall. $\delta=-2 \varphi^{\prime} / 3$.

surface du massif restant sous l'excavation, pour ne pas provoquer de soulèvement parasite du terrain. Cette opération a pour objectif de dégager l'écran pour les représentations graphiques, sans modifier l'état du sol dessous ; 
- augmentation de la pression sur l'écran par étapes jusqu'à la rupture (divergence du calcul de déformations). Dans certains cas, le calcul converge et s'arrête avant d'atteindre la rupture (ce qui est possible de la part d'une borne inférieure). Dans ce cas, on a réduit c et $\varphi$ pour définir les conditions de rupture, donc la pression correspondant à $\mathrm{K}_{\mathrm{p}}$.

Les déplacements associés à la rupture sont compris entre 50 et $180 \mathrm{~mm}$. Ces valeurs sont compatibles avec les déplacements considérés comme nécessaires pour mobiliser de la butée en fonction de la hauteur libre $\mathrm{H}$ de l'écran (de l'ordre de $\mathrm{H} / 100$, soit environ $100 \mathrm{~mm}$ pour $\mathrm{H}=10 \mathrm{~m}$ ).

\subsubsection{Développement libre du mécanisme de butée}

Cet exemple (Fig. 17) correspond à la déformation du sol quand on applique sur l'écran une pression horizontale proportionnelle à la profondeur (sans composante verticale, donc sans inclinaison). Cette pression part de $\mathrm{K}_{\mathrm{o}}$ et augmente jusqu'à la divergence du calcul. On en déduit la valeur du coefficient de butée $K_{p}$, soit $K_{P}=3,02$ (pour 3,00 dans les tables de Caquot-Kérisel-Absi et la même valeur pour le

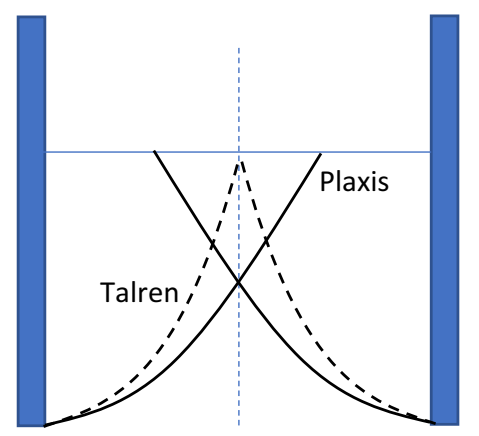

Fig. 16. Comparaison des surfaces de rupture de Talren et Plaxis.

Fig. 16. Comparison of Talren and Plaxis slip sufaces. mécanisme de Coulomb). Cette vérification sert à montrer que les calculs en éléments finis et la méthode de Caquot sont équivalents quand les conditions de validité de la méthode de Caquot sont satisfaites. Elle sert également à valider la méthodologie conformément au §5.1.3.4 (4) de la norme NF P94-282 (cohérence du modèle sur des cas comparables aux hypothèses de Kérisel et Absi).

\subsubsection{Développement du mécanisme de butée dans un espace limité par un autre écran}

On utilise le même modèle de calcul, mais un deuxième écran est placé à la distance $\mathrm{B}$ du premier. Dans le cas où $\mathrm{B}=\mathrm{D}$, on obtient le mécanisme de rupture de la figure 18 , avec $\mathrm{K}_{\mathrm{P}}=3,53$, soit une augmentation de $15 \%$ de la butée mobilisable. Ce coefficient est proche de celui calculé par Talren.

Le graphique de la figure 19 présente, pour le cas analysé ci-dessus, la variation du coefficient de butée en fonction de la distance entre les écrans (Jassionnesse et al., 2016) pour deux angles de frottement interne $\left(\varphi^{\prime}=30\right.$ degrés et $\varphi^{\prime}=35$ degrés) et pour deux inclinaisons de la force de butée $\left(\delta_{p}=0\right.$ et $\left.\delta_{\mathrm{p}}=-2 \varphi^{\prime} / 3\right)$ pour $\varphi=30$ degrés. L'effet de la présence de l'écran parallèle sur la butée mobilisable est visible pour $\mathrm{B} / \mathrm{D}<2$. Ces valeurs peuvent être directement utilisées dans les calculs de type MISS pour corriger l'estimation du coefficient de butée par les abaques de Caquot-Kérisel-Absi.

\section{Influence du mode d'évaluation de la sécurité}

Nous nous intéressons maintenant à l'évaluation de la sécurité sur la butée uniquement, par un calcul en éléments finis. L'intérêt particulier porté à la butée est lié à la façon dont la poussée et la butée sont traitées dans le dimensionnement des ouvrages. On considère la poussée, qui se développe rapidement, comme une action, tandis que la butée, qui se

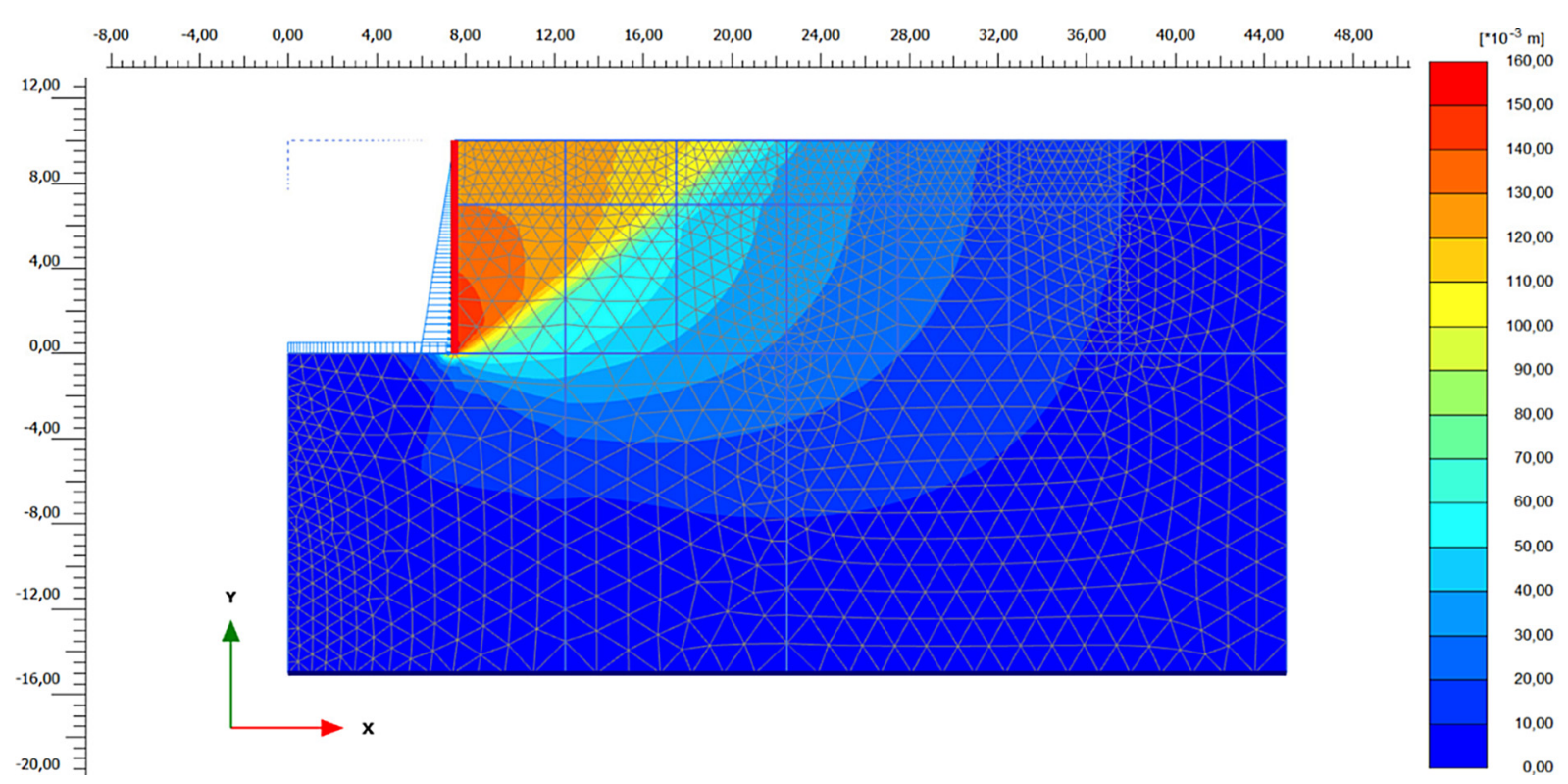

Fig. 17. Calcul en éléments finis de la butée du sol (écran isolé). Déplacement total.

Fig. 17. Finite element calculation of passive pressure (isolated embedded wall). Total displacement. 


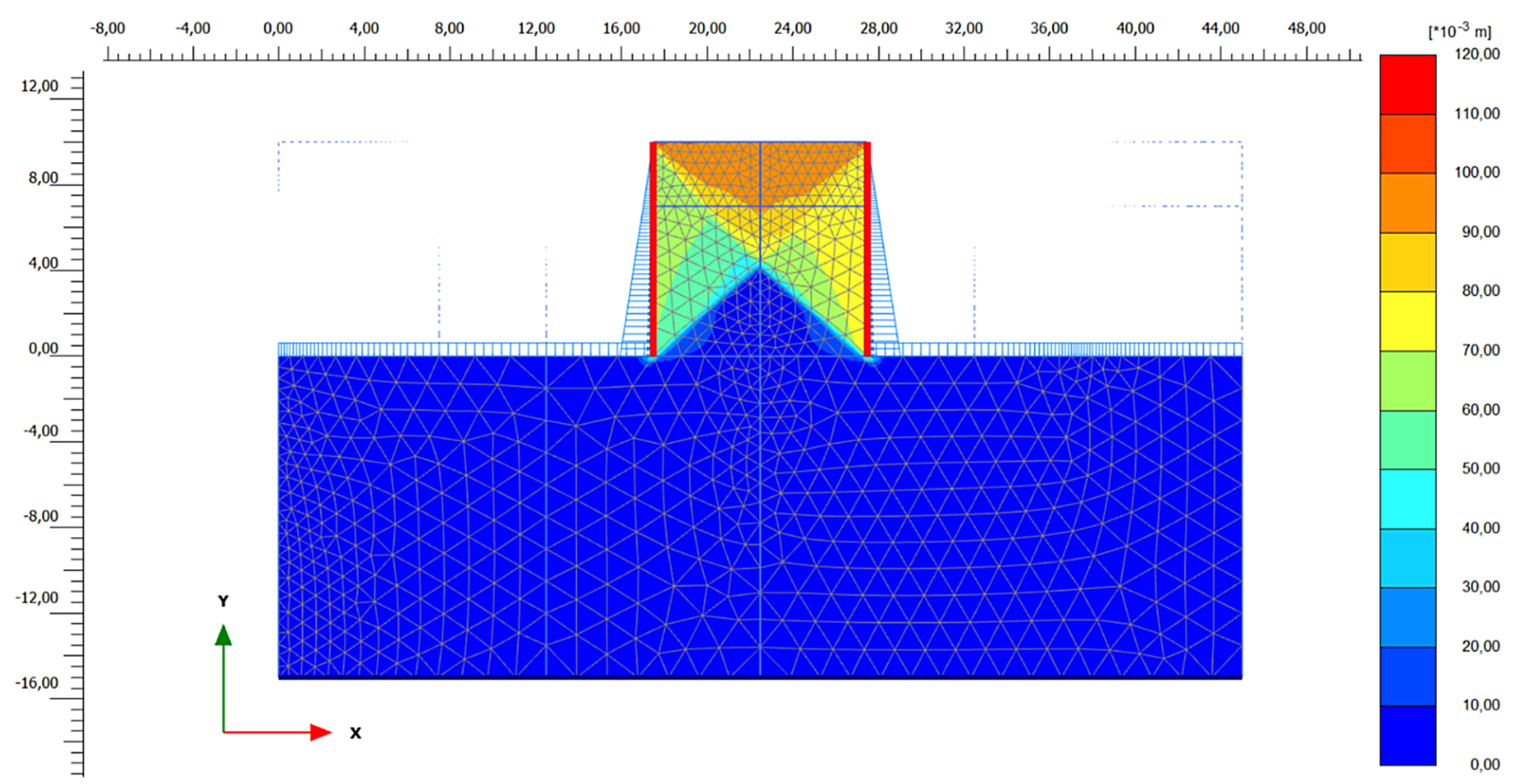

Fig. 18. Calcul en éléments finis de la butée du sol (deux écrans parallèles. $B / D=1$ ). Déplacement total.

Fig. 18. Finite element calculation of passive pressure (two parallel embedded walls $B / D=1$ ). Total displacement.

\section{Evolution du coefficient de butée en fonction de la distance entre} deux écrans $\left(\delta / \varphi^{\prime}=-2 / 3\right)$

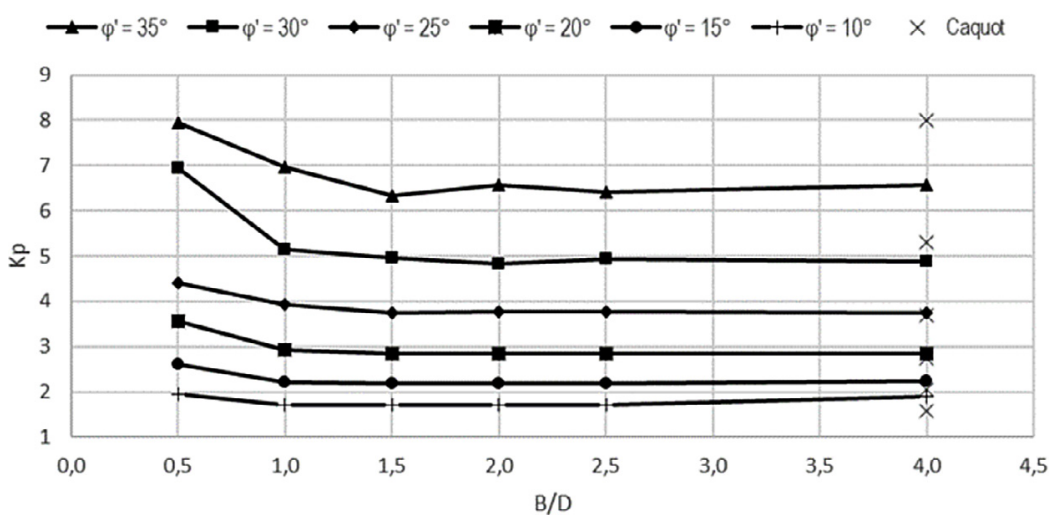

\begin{tabular}{|c|c|c|c|c|c|c|}
\hline$\beta / \varphi^{\prime}=0,00$ & $\delta / \varphi^{\prime}=-0,66$ & $\lambda=0,00$ & & & & \\
\hline $\mathrm{B} / \mathrm{D} \quad \varphi^{\prime}$ & 10 & 15 & 20 & 25 & 30 & 35 \\
\hline 4,0 & 1,72 & 2,25 & 2,84 & 3,74 & 4,87 & 6,56 \\
\hline 2,5 & 1,72 & 2,18 & 2,84 & 3,76 & 4,94 & 6,41 \\
\hline 2,0 & 1,70 & 2,18 & 2,84 & 3,76 & 4,84 & 6,57 \\
\hline 1,5 & 1,70 & 2,18 & 2,85 & 3,74 & 4,97 & 6,34 \\
\hline 1,0 & 1,71 & 2,22 & 2,94 & 3,92 & 5,15 & 6,96 \\
\hline 0,5 & 1,94 & 2,62 & 3,57 & 4,42 & 6,94 & 7,95 \\
\hline
\end{tabular}

Fig. 19. Évolution de $K_{p}$ avec la distance entre deux écrans parallèles.

Fig. 19. Evolution of $\mathrm{K}_{\mathrm{p}}$ with the distance between two parallel embedded walls.

développe pour des déformations plus importantes, est une réaction, qui est mobilisée autant que de besoin. On doit donc comparer la butée mobilisée pour assurer l'équilibre à la butée mobilisable, comme demandé notamment par la norme de calcul des écrans NF P94-282.

\subsection{Limites de la réduction de c' et $\varphi$ '}

La méthode de réduction de c' et $\varphi_{-}^{\prime}$ mise en œuvre dans Plaxis sur l'ensemble du massif de sol des deux côtés de l'écran a pour effet de dégrader l'équilibre en modifiant à la 


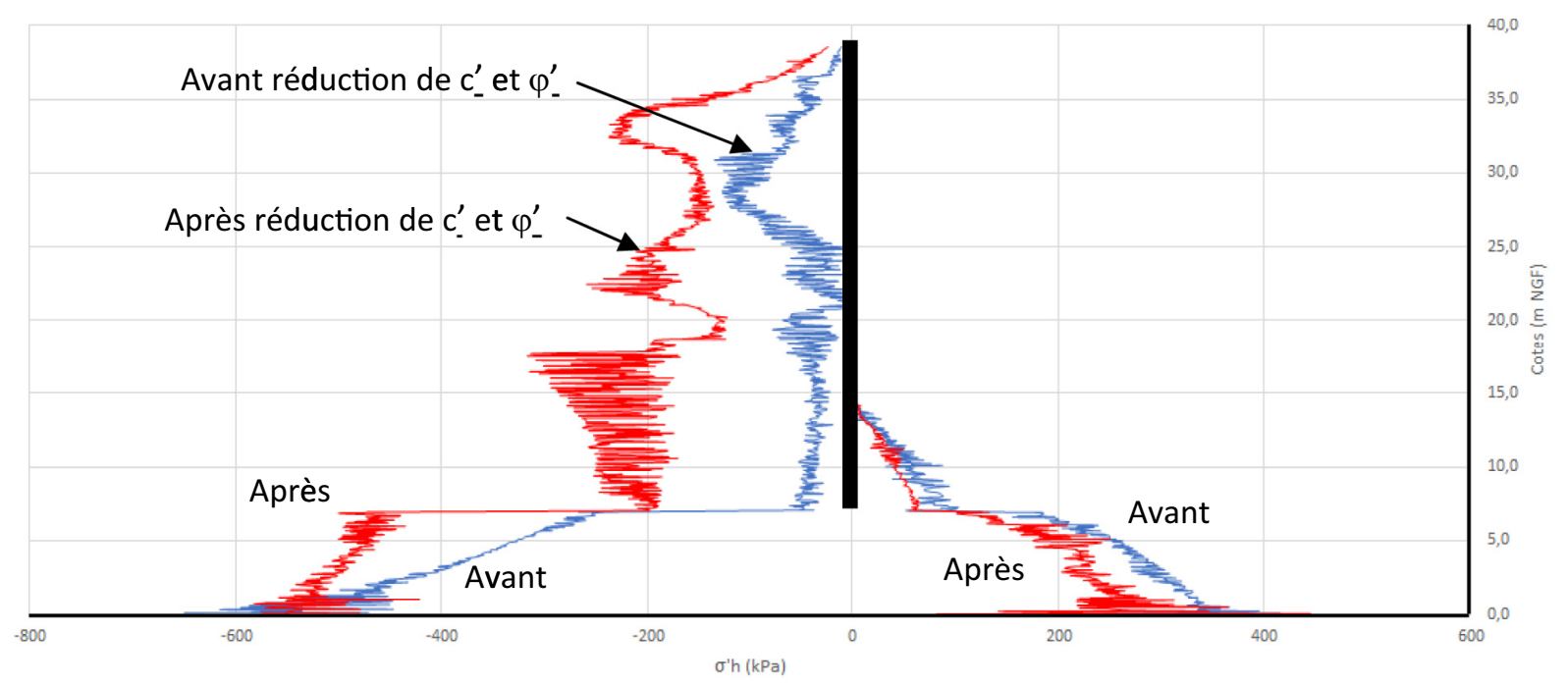

Fig. 20. Évolution de la contrainte horizontale en fonction de la profondeur, lors de l'opération de réduction de c' et $\varphi_{-}$.

Fig. 20. Evolution of horizontal stress with depth, due to c' and $\varphi_{-}^{\prime}$ reduction.

fois la poussée et la butée. Cette évolution des pressions sur un écran du fait de la dégradation générale de c' et $\varphi$ ' est illustrée par la figure 20 . On voit que la réduction de la cohésion et de la tangente de l'angle de frottement interne dans tout le maillage augmente beaucoup la poussée et diminue un peu la butée.

Or la norme, qui s'appuie sur l'expérience classique du dimensionnement des écrans, considère que la poussée est constante et qu'il faut évaluer de combien on peut augmenter la pression sur le sol jusqu'à la rupture en butée. La réduction générale de c' et $\varphi_{-}^{\prime}$ ne fournit pas la valeur de butée mobilisable au-delà de l'équilibre actuel et ne peut être recommandée. Par contre, les valeurs représentées sur la figure 19 restent valables, puisqu'elles ne font pas intervenir la poussée.

\subsection{Procédure suggérée pour évaluer la sécurité sur la butée}

Une réflexion a donc été menée pour le cas des ouvrages réels, en cherchant à intégrer dans le calcul de la butée mobilisable les conditions géométriques imposées aux écrans, notamment par les butons et les dalles. Il a été choisi de distinguer dans le maillage de calcul la zone où se développera la butée, située sous le fond de fouille, et de lui attribuer des propriétés dégradées (Chapron et al., 2016).

Dans cette zone du maillage, on divise directement les paramètres c' (ou c $\mathrm{u}_{\mathrm{u}}$ pour les conditions non drainées) et tan $\varphi$ ' par le coefficient de sécurité global recherché, afin de tester uniquement la butée, tous les autres paramètres gardant leur valeur initiale. La valeur du coefficient de sécurité en phase transitoire est fixée à 1,485 par la norme NF P94-282. La vérification à long terme, lorsque toute la structure est finie, notamment les radiers, s'effectue avec un coefficient de sécurité de 1,9 .

La zone dont les paramètres de résistance ont été modifiés est représentée sur la figure 21 . Le calcul a convergé, preuve que la butée est suffisante (Utter, 2017) avec un facteur de sécurité d'au moins 1,5 (coefficient arrondi utilisé pour le calcul, à la place de 1,485). Ce n'est plus le cas si l'on divise les paramètres de résistance au cisaillement par 2 dans la zone de butée. La procédure numérique ne converge plus à partir de $95 \%$ du calcul, ce qui suggère que le coefficient de sécurité est compris entre 1,5 et 2 . La figure 22 présente les résultats de ce second calcul.

La même démarche de calcul, appliquée à un écran isolé en réduisant les paramètres de résistance c' et tan $\varphi$ ' d'un facteur 1,5 , ne converge que si l'on prolonge la fiche de l'écran de $4 \mathrm{~m}$.

\subsection{Choix du coefficient de sécurité sur les paramètres de cisaillement}

Le choix des facteurs de réduction de la résistance au cisaillement dans les calculs pour obtenir un effet global de 1,5 (ou plus précisément 1,485 en phase transitoire) mérite une analyse détaillée. Le calcul analytique de la butée se traduit par une valeur de pression $\mathrm{B}_{\mathrm{t} ; \mathrm{d}}$ égale à :

$-\mathrm{B}_{\mathrm{t} ; \mathrm{d}}=\mathrm{K}_{\mathrm{p}} \sigma^{\prime}{ }_{\mathrm{vo}}+2 \mathrm{c}^{\prime} \sqrt{\mathrm{K}_{\mathrm{p}}}$, en conditions drainées;

$-\mathrm{B}_{\mathrm{t} ; \mathrm{d}}=\sigma_{\mathrm{vo}}+2 \mathrm{c}_{\mathrm{u}}$, en conditions non drainées.

La pression de l'eau est gérée séparément, sans facteur de sécurité spécifique.

La valeur du coefficient de butée est calculée en utilisant la formule $K_{P}=\tan ^{2}\left(\pi / 4+\varphi^{\prime} / 2\right)$ dans le cas d'un écran vertical, d'un talus horizontal et d'une inclinaison nulle de la butée. Dans les autres cas, les équations de Caquot-Kérisel doivent être résolues. Dans la suite du paragraphe, seul le cas d'un écran vertical et d'un talus horizontal est traité et les paramètres de résistance au cisaillement à intégrer dans un calcul aux éléments finis afin de retrouver une sécurité sur la butée de 1,485 en phase provisoire sont indiqués.

\subsubsection{Cas d'un sol purement frottant $\left(c^{\prime}=0 \mathrm{kPa}\right)$}

Dans ce cas, la relation entre le facteur de portance et la butée mobilisable est proportionnelle et ne dépend que de l'angle $\varphi$ ' par l'intermédiaire de $K_{\mathrm{p}}$. En fonction de l'inclinaison de la butée, la dégradation de l'angle de 


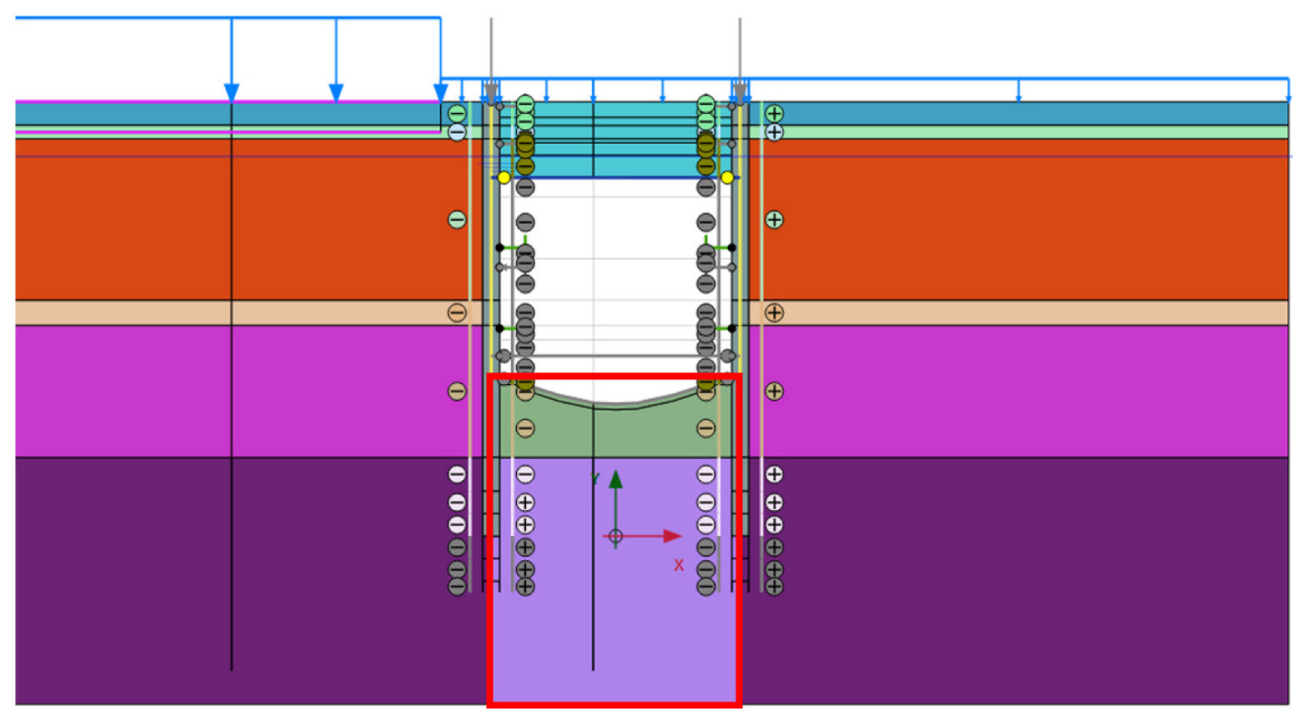

Fig. 21. Géométrie de la zone en butée à caractéristiques minorées (sous le fond de fouille).

Fig. 21. Geometry of the passive zone with lower strength under the bottom of the excavation.

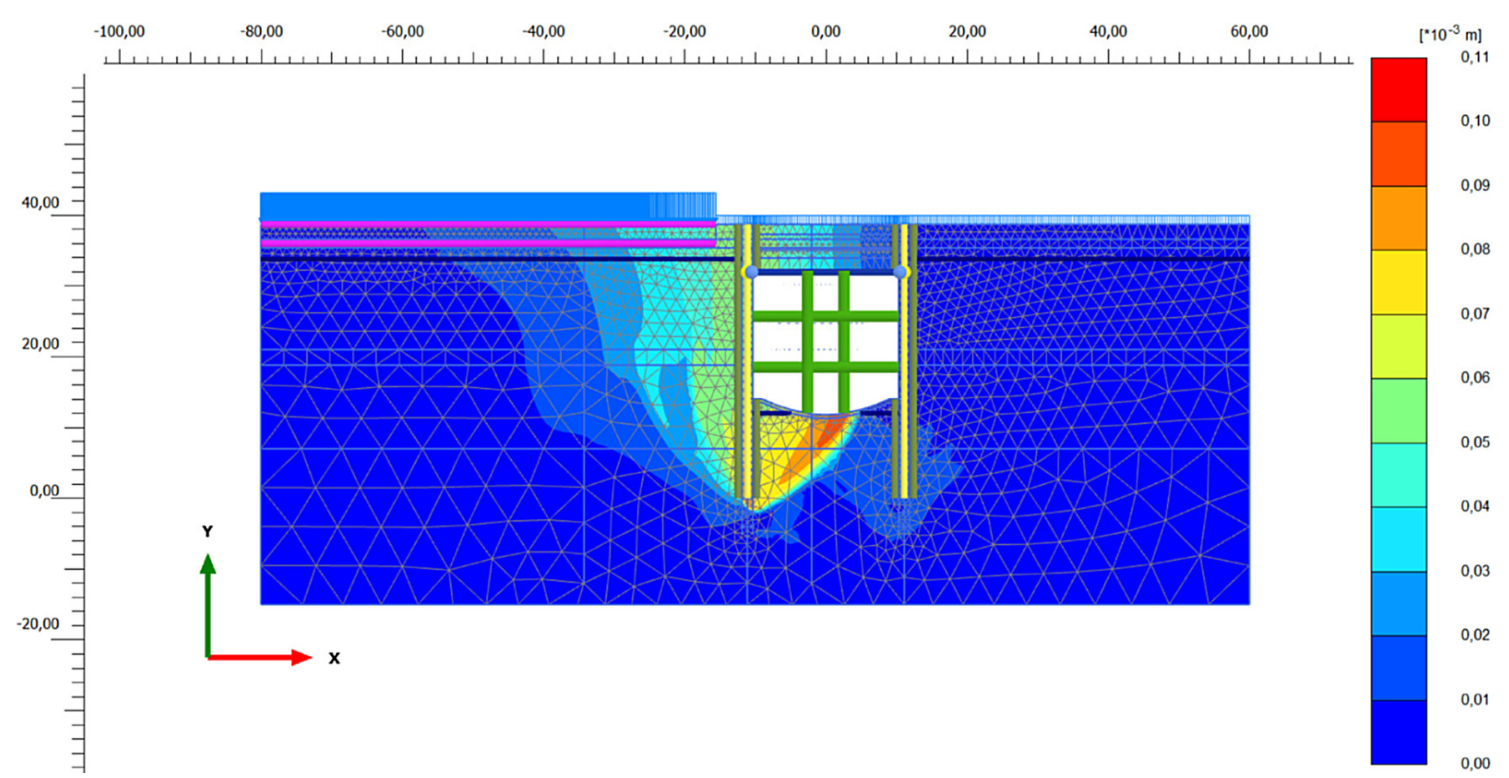

Fig. 22. Incréments de déplacements calculés à la fin du chargement (paramètres de cisaillement divisés par 2 dans la zone de butée). Calcul arrêté à $95 \%$.

Fig. 22. Incremental displacements at the end of calculations (stopped at 95\%) for strengths divided by a factor 2 .

frottement peut être déduite du graphique de la figure 23 et du tableau 2, qui permettent de retrouver une sécurité de 1,485 sur la butée.

\subsubsection{Cas d'un sol frottant et cohérent $\left(c^{\prime} \neq 0 \mathrm{kPa}\right)$}

Compte tenu de la formule de calcul de la butée mobilisable $\mathrm{B}_{\mathrm{t} ; \mathrm{d}}$, l'application d'un coefficient de sécurité de 1,485 revient à diviser chaque terme par ce coefficient. Le premier terme a été traité précédemment et fixe donc l'angle de frottement dégradé dans le cas d'un sol purement frottant. Pour un sol frottant et cohérent, l'analyse reste identique pour le terme de frottement $\left(\mathrm{K}_{\mathrm{P}}\right)$ (Tab. 2). Pour le terme cohérent et Q4 frottant $\left(2 \mathrm{c}^{\prime} \sqrt{\mathrm{K}_{\mathrm{p}}}\right)$, le facteur $\sqrt{\mathrm{K}_{\mathrm{p}}}$ est fixé par l'analyse sans cohésion. La cohésion dégradée est donc déterminée en fonction de la sécurité manquante sur $\sqrt{\mathrm{K}_{\mathrm{p}}}$. D'où une dégradation de la cohésion de $\sqrt{1,485}=1,219$ en plus des paramètres retenus pour un sol purement frottant (Tab. 3).

\subsubsection{Cas d'un sol purement cohérent $\left(c_{u} \neq 0\right.$, $\left.\varphi_{u}=0\right)$ - sols non drainés}

L'application d'un facteur global à la butée calculée en contraintes totales pose un problème car cela signifie que 
l'on divise par 1,485 non seulement la résistance due à la cohésion mais aussi la contrainte totale verticale, que l'on n'a pas de raisons de remettre en question. La solution impliquée par les règles de l'Eurocode 7 serait de diviser la cohésion non drainée par un facteur partiel égal à 1,4 pour obtenir sa valeur de calcul à l'ELU. Cette vérification, applicable aux situations temporaires, sera complétée par une vérification à long terme sur la butée calculée en contraintes effectives.
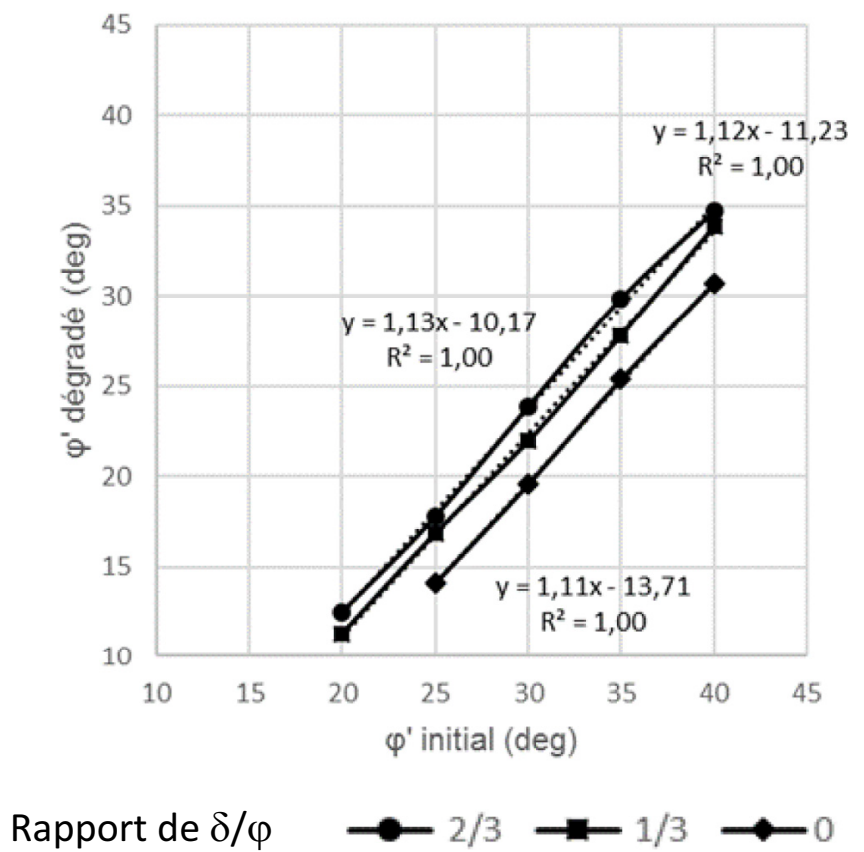

Fig. 23. Relation entre les angles $\varphi$ ' initial et $\varphi$ ' dégradé pour obtenir une réduction de $\mathrm{K}_{\mathrm{p}}$ de 1,485 .

Fig. 23. Relation between the initial friction angle and the lowered one to have $\mathrm{K}_{\mathrm{p}}$ decreased by a factor 1.485 .

\section{Conclusions}

Les analyses présentées dans cet article montrent que la méthode de Caquot ne peut pas toujours être utilisée pour évaluer la butée mobilisable dans le calcul des écrans en vis-àvis. Cette conclusion est importante pour les calculs d'écrans par la méthode des coefficients de réaction, où l'on calcule l'équilibre d'une poutre sur appuis élastiques entre la poussée, la butée et la réaction des appuis. Il est admis que la poussée se développe d'abord et que l'équilibre mobilise la butée autant que de besoin, de sorte que l'on cherche à comparer la butée déjà mobilisée par l'équilibre avec la butée mobilisable. Cette comparaison doit s'effectuer à poussée constante, puisque la butée mobilisée est définie pour équilibrer cette poussée. Les calculs à la rupture et aux éléments finis montrent que la distance entre deux écrans proches influe sur la butée mobilisable de ces ouvrages, quand le rapport B/D devient inférieur à 2.

Pour évaluer la marge de stabilité, écart entre la butée mobilisable et la butée mobilisée, la méthode de réduction de $\mathrm{c}$ et $\varphi$ (c' et $\varphi$ ' ou c ${ }_{\mathrm{u}}$, selon les cas) utilisée dans le logiciel Plaxis, qui s'applique à la totalité des terrains du maillage, modifie complètement le mécanisme de déformation du sol et notamment la poussée. Elle ne peut pas être appliquée pour évaluer la butée mobilisable au-delà de la butée mobilisée dans le calcul. En revanche, la limitation de la zone où l'on réduit la résistance du sol fournit des résultats utiles.

Tableau 3. Cohésion dégradée afin d'obtenir une butée mobilisable divisée par 1,485.

Table 3. Lowered cohesion to obtain a passive resistance divided by 1.1485 .

\begin{tabular}{lllllll}
\hline & \multicolumn{6}{c}{ c' initial $(\mathrm{kPa})$} \\
\cline { 2 - 7 } & 5 & 10 & 15 & 20 & 25 & 30 \\
\hline c' dégradé $(\mathrm{kPa})$ & 4,1 & 8,2 & 12,3 & 16,4 & 20,5 & 24,6 \\
Rapport & 1,219 & 1,219 & 1,219 & 1,219 & 1,219 & 1,219 \\
\hline
\end{tabular}

Tableau 2. Angle de frottement dégradé afin d'obtenir une butée divisée par 1,485.

Table 2. Lowered friction angle to obtain a passive resistance divided by 1.485 .

\begin{tabular}{|c|c|c|c|c|c|c|c|c|}
\hline \multicolumn{9}{|c|}{ Cas d'un sol purement frottant $\left(\mathrm{c}^{\prime}=0 \mathrm{kPa}\right)$} \\
\hline \multicolumn{3}{|c|}{ Inclinaison de la butée $\delta_{\mathrm{p}} / \varphi^{\prime}=0$} & \multicolumn{3}{|c|}{$\underline{\text { Inclinaison de la butée } \delta_{\mathrm{p}} / \varphi^{\prime}=1 / 3}$} & \multicolumn{3}{|c|}{ Inclinaison de la butée $\delta_{\mathrm{p}} / \varphi^{\prime}=2 / 3$} \\
\hline $\begin{array}{l}\text { Angle de } \\
\text { frottement } \\
\text { initial }\end{array}$ & $\begin{array}{l}\text { Angle de } \\
\text { frottement } \\
\text { dégradé }\end{array}$ & $\begin{array}{l}\text { Ratio sur la } \\
\text { butée }\end{array}$ & $\begin{array}{l}\text { Angle de } \\
\text { frottement } \\
\text { initial }\end{array}$ & $\begin{array}{l}\text { Angle de } \\
\text { frottement } \\
\text { dégradé }\end{array}$ & $\begin{array}{l}\text { Ratio sur la } \\
\text { butée }\end{array}$ & $\begin{array}{l}\text { Angle de } \\
\text { frottement } \\
\text { initial }\end{array}$ & $\begin{array}{l}\text { Angle de } \\
\text { frottement } \\
\text { dégradé }\end{array}$ & $\begin{array}{l}\text { Ratio sur } \\
\text { la butée }\end{array}$ \\
\hline 20,0 & - & - & 20,0 & 11,3 & 1,485 & 20,0 & 12,5 & 1,485 \\
\hline 25,0 & 14,1 & 1,485 & 25,0 & 16,9 & 1,485 & 25,0 & 17,8 & 1,485 \\
\hline 30,0 & 19,6 & 1,485 & 30,0 & 22,0 & 1,485 & 30,0 & 23,9 & 1,485 \\
\hline 35,0 & 25,4 & 1,485 & 35,0 & 27,8 & 1,485 & 35,0 & 29,9 & 1,485 \\
\hline 40,0 & 30,7 & 1,485 & 40,0 & 33,9 & 1,485 & 40,0 & 34,7 & 1,485 \\
\hline
\end{tabular}


La méthode retenue pour évaluer la marge de sécurité consiste à définir une zone de terrain devant l'écran qui aura une résistance au cisaillement réduite. Les propriétés de cette zone seront réduites d'un facteur 1,485 (coefficient de la norme NF P94-282 en phase provisoire, mais on peut faire le même calcul avec un facteur de 1,9 dans les conditions d'équilibre à long terme) et l'on calculera les déformations de l'ouvrage avec ces valeurs de c' et $\varphi^{\prime}$ ou $c_{\mathrm{u}}$, selon les cas. Si le calcul converge vers un champ de déformations, on conclut que la butée mobilisable est supérieure d'un facteur au moins égal à 1,485 à la butée mobilisée, tel que demandé dans la norme. On vérifie en complément que les forces reprises par les butons et les sollicitations dans la structure restent acceptables (inférieures à l'ELU).

Cette méthode peut être utilisée dans le cas d'un écran isolé comme dans le cas de deux écrans parallèles. On peut de cette façon définir pour des cas simples d'écrans en interaction des coefficients de butée complémentaires à ceux des tables de Kérisel et Absi (2003).

\section{Références}

Absi É. 1984. La théorie de la plasticité et l'équilibre limite en mécanique des sols. Paris: Annales de l'ITBTP, $n^{\circ} 421$, Série Sols et Fondations 185, pp. 65-123.

Boussinesq JV. 1876. Essai théorique sur l'équilibre d'élasticité des massifs pulvérulents et sur la poussée des terres sans cohésion (Mémoire présenté à la classe des sciences dans la séance du 6 juin 1874). Extrait du tome XL des «Mémoires couronnés et Mémoires des savants étrangers $»$. Académie royale des sciences, des lettres et des beaux-arts de Belgique, $\mathrm{n}^{\mathrm{o}} 4$. Bruxelles: Imprimerie de $\mathrm{F}$. Hayez, 180 p. Disponible sur http://iris.univ-lille1.fr/handle/1908/ 1398.

Bresse JAC. 1859. Cours de mécanique appliquée professé à l'École Impériale des Ponts et Chaussées. Première partie : résistance des matériaux et stabilité des constructions. Paris: Mallet-Bachelier, Imprimeur-Libraire, $470 \mathrm{p}$.

Caquot A. 1934. Équilibre des massifs à frottement interne. Stabilité des terres pulvérulentes ou cohérentes. Paris: Gauthier-Villars, Éditeur, 92 p.

Chapron G, Nejjar K, Le Bissonnais H, Cuira F. 2016. Considérations pratiques autour de modèles numériques de certaines gares du futur Grand Paris, JNGG 2016.

Corradi M. De la statique des demi-fluides à la théorie de la poussée des terres. In: Radelet de Grave P, Benvenuto E, eds. Entre
Mécanique et Architecture/Between Mechanics and Architecture. Springer Science \& Business Media, 20 déc. 1994, 404 p. pp. 221-256. Basel: Birkhaüser Verlag, 1995, 399 p.

Coulomb CA. 1773. Essai sur une application des règles de maximis et de minimis à quelques problèmes de statique relatifs à l'architecture. Paris: Académie Royale des Sciences. Mémoires de mathématique et de physique par divers savants, Vol. 7, pp. 343-382.

Culmann K. 1866. Die graphische Statik. Zürich: Verlag Von Meyer \& Zeller, 634 p. + figures.

Droniuc N, Magnan JP. 2010. Travaux sur le calcul des soutènements par l'analyse limite. Paris: Laboratoire Central des Ponts Chaussées, non publié.

Jassionnesse C, Cahn M, Tsirogianni A. 2016. Interaction butée des rideaux en vis-à-vis. JNGG 2016.

Kérisel J, Absi É. 2003. Tables de poussée et de butée des terres. Paris : Éditions Presses ENPC, 3 édition.

Mokhbi H, Messaset S. 2014. Étude numérique de la poussée et de la butée des terres. Courr. Savoir 18: 31-40.

Montès P. 2012. Poussées, butées, murs de soutènement : liens vers les travaux de Coulomb (1773), de Poncelet (1840), de Culmann (1866), de Rebhann (1871), etc., mis à jour le samedi 14 avril 2012. Disponible sur http://jfjpm-genie-civil.blogspot.fr/.

Müller-Breslau HFB. 1881. Éléments de statique graphique appliquée aux constructions, Vol. 1. Traduit de l'allemand par T. Seyrig. Paris: Librairie polytechnique Baudry et Cie., 1886, 407 p.

NAVFAC DM-7.2. 1982. Foundations and earth-structures. Design manual. USA: Department of the Navy.

Norme NF EN 1997-1. 2004. Eurocode 7. Calcul géotechnique. AFNOR.

Norme NF P94-282. 2009. Calcul géotechnique, Ouvrages de soutènement. AFNOR.

Philipponnat G, Hubert B. 1997. Fondations et ouvrages en terre. $4^{\mathrm{e}}$ édition. Paris : Eyrolles, 576 p.

Poncelet M. 1840. Mémoire sur la stabilité des revêtements et de leurs fondations. Mémorial de l'Officier du Génie, $\mathrm{n}^{\mathrm{o}} 13$. Paris: Imprimerie et Librairie de Bachelier, pp. 7-270.

Rankine WJM. 1857. On the stability of loose earth. London: Philosophical Transactions of the Royal Society of London, Vol. 147.

Rebhann G. 1871. Theorie des Erddruckes und der Futtermauern. Wien: Druck und Verlag von Carl Gerold's Sohn, 544 p.

Résal J. 1903. Poussée des terres. Stabilité des murs de soutènement. Cours de l'École des Ponts et Chaussées. Paris: Librairie Polytechnique, Ch. Béranger. Éditeur, 254 p.

Utter N. 2017. Sécurité sur la butée des écrans de soutènement: quelques cas particuliers. Rev Trav 933: 46-51.

Citation de l'article : Jean-Pierre Magnan, Grégory Meyer. Influence des interactions entre écrans de soutènement sur le calcul de la butée. Rev. Fr. Geotech. 2018, 154, 2. 